\title{
HECKE-TYURIN PARAMETRIZATION OF THE HITCHIN AND KZB SYSTEMS
}

\author{
B. ENRIQUEZ AND V. RUBTSOV
}

\begin{abstract}
We study the parametrization of the moduli space $\operatorname{Bun}_{2}(C)_{\mathcal{L}}$ of rank 2 bundles over a curve $C$ with fixed determinant, provided by Hecke modifications at fixed points of the trivial bundle. This parametrization is closely related to the Tyurin parametrization of vector bundles over curves. We use it to parametrize the Hitchin and KZB systems, as well as lifts of the Beilinson-Drinfeld $\mathcal{D}$-modules. We express a generating series for the lifts of the Beilinson-Drinfeld operators in terms of a "quantum $L$-operator" $\ell(z)$. We explain the relation to earlier joint work with G. Felder, based on parametrization by flags of bundles, and introduce filtrations on conformal blocks, related with Hecke modifications.
\end{abstract}

\section{INTRODUCTION}

1.1. Tyurin maps and Hecke parametrizations. In [22], A. Tyurin introduced the following parametrization of the moduli space $\operatorname{Bun}_{n}(C)_{n g}$ of vector bundles of rank $n$ and of degree $n g$ over a curve $C$ of genus $g=g(C) \geq 1$. The space $H^{0}(C, \mathcal{E})$ of sections of a generic such bundle $\mathcal{E}$ is of dimension $n$. For $P$ a point of $C$, denote by $\mathcal{E}_{P}$ the fiber of $\mathcal{E}$ at $P$ and define $\Sigma_{P}$ as the subspace of $\mathcal{E}_{P}$ generated by the sections of $\mathcal{E}$. $\Sigma_{P}$ is equal to the fiber of $\mathcal{E}_{P}$ at $P$, except when $P$ is one of $n g$ points of $C, P_{1}, \ldots, P_{n g}$. Generically, the $P_{i}$ are distinct and $\Sigma_{P}$ is of codimension 1 in $\mathcal{E}_{P}$. Let us denote by $\ell_{i}$ the subspace of $H^{0}(C, \mathcal{E})^{*}$ generated by evaluation at $P_{i}$. Define $\mathcal{T}$ as the map assigning to $\mathcal{E}$, the collection $\left(P_{i}\right)_{i=1, \ldots, n g}$ together with the collection of lines $\left(\ell_{i}\right)_{i=1, \ldots, n g}$ in $H^{0}(C, \mathcal{E})^{*}$, modulo diagonal action of $S L_{n}(\mathbb{C})$. The resulting map

$$
\mathcal{T}: \operatorname{Bun}_{n}(C)_{n g} \rightarrow\left[C \times \mathbb{P}\left(\mathbb{C}^{n}\right)\right]^{(n g)} / S L_{n}(\mathbb{C})
$$

is called the Tyurin map (we denote by $V^{(N)}$ the $N$ th symmetric power of a variety $V$ ). After its introduction in [22], the Tyurin map was used in works on integrable differential systems (see [13, 17, 19]).

The inverse of the map $\mathcal{T}$ can be naturally described in terms of Hecke modifications. For $\mathcal{E}$ a rank $n$ vector bundle over $C, \underline{P}=\left(P_{i}\right)_{i=1, \ldots, N}$ a family of points of $C$ and $\underline{V}=\left(V_{i}\right)_{i=1, \ldots, N}$ a family of vector subspaces of $\mathcal{E}_{P_{1}}, \ldots, \mathcal{E}_{P_{N}}$, define the Hecke modification $\mathcal{H}(\mathcal{E}, \underline{P}, \underline{V})$ of $\mathcal{E}$ as the bundle whose space of sections over an open subset $U \subset C$ is the space of rational sections of $\mathcal{E}$ over $U$, regular

Date: November 1999. 
except at the points $P_{i}$, where they may have simple poles, with residue in $V_{i}$. In the case where $\mathcal{E}$ is the trivial bundle $\mathcal{O}_{C}^{n}$, and $V_{i}$ are lines $\ell_{i}$ in $\mathbb{C}^{n}$, let us set $\underline{\ell}=\left(\ell_{i}\right)_{i=1, \ldots, N}$ and $\mathcal{H}(\underline{P}, \underline{\ell})=\mathcal{H}\left(\mathcal{O}_{C}^{n}, \underline{P}, \underline{\ell}\right)$. Since the automorphism group of $\mathcal{O}_{C}^{n}$ is equal to $G L_{n}(\mathbb{C})$, the map

$$
\mathcal{H}:\left(C \times \mathbb{P}\left(\mathbb{C}^{n}\right)\right)^{(N)} \rightarrow \operatorname{Bun}_{n}(C)
$$

factors through the diagonal action of $G L_{n}(\mathbb{C})\left(\right.$ or $\left.S L_{n}(\mathbb{C})\right)$. In the case where $N=n g$, the resulting map (also denoted $\mathcal{H}$ ) is inverse to the Tyurin map.

In the case $n=1$, the map $\mathcal{H}$ coincides with the Abel-Jacobi map from the symmetric powers of $C$ to its Picard variety. It is therefore natural to view $\mathcal{H}$ as a nonabelian analogue of the Abel-Jacobi map.

1.2. Main results. In this paper, we will keep the points $\underline{P}=\left(P_{i}\right)_{i=1, \ldots, n g}$ fixed, and associate to them the map $\mathcal{H}_{\underline{P}}$ such that $\mathcal{H}_{\underline{P}}(\underline{\ell})=\mathcal{H}(\underline{P}, \underline{\ell})$. We will study $\mathcal{H}_{\underline{P}}$ (sect. 2) and parametrize in terms of it natural objects attached to the moduli spaces of vector bundles, in the case $n=2$. These objects are the Hitchin system (sect. 3), the bundle of conformal blocks (sect. 1), the Beilinson-Drinfeld $\mathcal{D}$-modules (sect. 6) and the Knizhnik-Zamolodchikov-Bernard (KZB) connection

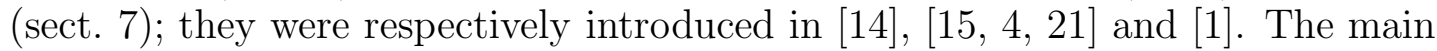
results of this paper are the following.

1.2.1. Properties of $\mathcal{H}_{\underline{P}}$. Let us set $N=3 g, G=S L_{2}$. $\mathcal{H}_{\underline{P}}$ is then a map from $\left(\mathbb{C} P^{1}\right)^{3 g} / G(\mathbb{C})$ from the moduli space $\operatorname{Bun}_{2}(C)_{\mathcal{O}\left(\sum_{i} P_{i}\right)}$ of rank 2 bundles with determinant isomorphic to $\mathcal{O}\left(\sum_{i} P_{i}\right)$.

Let us fix a choice of $a$ - and $b$-cycles $\left(A_{a}, B_{a}\right)_{a=1, \ldots, g}$ on $C$, and let $\left(\omega_{a}\right)_{a=1, \ldots, g}$ be the associated basis of regular differentials on $C$; we have $\int_{A_{a}} \omega_{b}=2 i \pi \delta_{a b}$.

Let us set

$$
\begin{aligned}
\operatorname{Den}(\underline{P}, \underline{\ell})= & \sum_{\sigma \in \mathfrak{S}_{3 g}: \sigma(3 a-2)<\sigma(3 a-1)<\sigma(3 a) \text { for } a=1, \ldots, g} \\
& \varepsilon(\sigma) \sigma \cdot\left\{\prod_{a=1}^{g} \omega_{a}\left(P_{3 a-2}\right) \omega_{a}\left(P_{3 a-1}\right) \omega_{a}\left(P_{3 a}\right) \ell_{3 a-2 ; 3 a-1} \ell_{3 a-2 ; 3 a} \ell_{3 a-1 ; 3 a}\right\},
\end{aligned}
$$

where $\ell_{i j}=\ell_{i}-\ell_{j}$, and the elements $\sigma$ of $\mathfrak{S}_{3 a}$ act by permuting both the $P_{i}$ and the $\ell_{i}$.

Theorem 1.1. The preimage of $\operatorname{Bun}_{2}(C)_{\mathcal{O}\left(\sum_{i} P_{i}\right)}^{\text {stable }}$ by $\mathcal{H}_{\underline{P}}$ contains the open $\{\underline{\ell} \in$ $\left.\left(\mathbb{C} P^{1}\right)^{3 g} \mid \operatorname{Den}(\underline{P}, \underline{\ell}) \neq 0\right\} / G(\mathbb{C})$. Moreover, the restriction of $\mathcal{H}_{\underline{P}}$ to

$$
\left\{\ell \in\left(\mathbb{C} P^{1}\right)^{3 g} \mid \operatorname{Den}(\underline{P}, \underline{\ell}) \neq 0\right\} / G(\mathbb{C})
$$

is an etale morphism from this variety to its image, which is a dense subset of $\operatorname{Bun}_{2}(C)_{\mathcal{O}\left(\sum_{i} P_{i}\right)}^{\text {stable }}$ 
HECKE-TYURIN PARAMETRIZATION OF THE HITCHIN AND KZB SYSTEMS

1.2.2. Parametrization of the Hitchin system. By symplectic reduction, we may identify $T^{*}\left[\left(\mathbb{C} P^{1}\right)^{3 g}\right] / G$ with the quotient of $\left\{(\underline{\ell}, \underline{\lambda}) \in \mathbb{C}^{3 g} \times \mathbb{C}^{3 g} \mid \sum_{i} \lambda_{i}=\sum_{i} \ell_{i} \lambda_{i}=\right.$ $\left.\sum_{i} \ell_{i}^{2} \lambda_{i}=0\right\} / G$ (see sect. 3$)$. Let $(e, f, h)$ be the Chevalley basis of $\overline{\mathfrak{g}}=\mathfrak{s l}_{2}$ and for $(\underline{\ell}, \underline{\lambda})$ in $\mathbb{C}^{3 g} \times \mathbb{C}^{3 g}$, let us set

$$
\begin{aligned}
& A(\underline{\ell}, \underline{\lambda} \mid z)=\sum_{i=1}^{3 g} \lambda_{i}\left(-e+\ell_{i} h+\ell_{i}^{2} f\right) \omega^{\left(P_{i}\right)}(z)+\frac{1}{\operatorname{Den}(\underline{P}, \underline{\ell})} \sum_{i, j=1, \ldots, 3 g ; j \neq i} \lambda_{i} \ell_{i j}^{2} \omega^{\left(P_{i}\right)}\left(P_{j}\right) \\
& \left\{\left.\frac{1}{2} \partial_{\ell_{j}}^{2} \operatorname{Den}(\underline{P}, \underline{\ell})\right|_{\ell_{j}=0} e+\frac{1}{2} \partial_{\ell_{j}} \operatorname{Den}(\underline{P}, \underline{\ell})_{\mid \ell_{j}=0} h-\operatorname{Den}(\underline{P}, \underline{\ell})_{\mid \ell_{j}=0} f\right\}_{\mid P_{j}=z},
\end{aligned}
$$

where $\ell_{i j}=\ell_{i}-\ell_{j}$ and

$$
\omega^{(z)}(P)=d_{P} \ln \Theta(A(P)-A(z)+\delta-\Delta)
$$

is the Green function on $C$ (see the conventions for theta-functions in sect. 2.1). $A(\underline{\ell}, \underline{\lambda} \mid z)$ is an element of $\overline{\mathfrak{g}} \otimes \Omega(C)$, where $\Omega(C)$ is the space of rational differentials on $C$. Let us set $H(\underline{\ell}, \underline{\lambda} \mid z)=\operatorname{tr} \rho[A(\underline{\ell}, \underline{\lambda} \mid z)]^{2}$.

The Hitchin Hamiltonians Hitch $\alpha$ are regular maps defined on $T^{*} \operatorname{Bun}_{2}(C)_{\mathcal{O}}\left(\sum_{i} P_{i}\right)$ (see sect. 3 and [14).

Proposition 1.1. $z \mapsto H(\underline{\ell}, \underline{\lambda} \mid z)$ is a regular quadratic differential on $C$. Let us set

$$
H(\underline{\ell}, \underline{\lambda} \mid z)=\sum_{\alpha=1}^{3 g-3} H_{\alpha}(\underline{\ell}, \underline{\lambda}) \omega_{\alpha}^{(2)}(z) .
$$

The $H_{\alpha}(\underline{\ell}, \underline{\lambda})$ are rational functions in $\ell_{i}$ and quadratic polynomials in $\lambda_{i}$. They are $G$-invariant functions on $\left\{(\underline{\ell}, \underline{\lambda})=\left(\ell_{i}, \lambda_{i}\right)_{i=1, \ldots, 3 g} \mid \sum_{i} \lambda_{i}=\sum_{i} \lambda_{i} \ell_{i}=\sum_{i} \lambda_{i} \ell_{i}^{2}=\right.$ $0\}$, and define therefore a family of functions on $T^{*}\left[\left(\mathbb{C} P^{1}\right)^{3 g} / G(\mathbb{C})\right]$. These functions form a Poisson-commutative family coinciding with the $\operatorname{Hitch}_{\alpha} \circ T^{*} \mathcal{H}_{\underline{P}}$.

1.2.3. Explicit form of the Beilinson-Drinfeld operators. The Beilinson-Drinfeld operators are a commuting family of globally defined differential operators $\left(T_{\alpha}^{B D}\right)_{\alpha=1, \ldots, 3 g-3}$ on $\operatorname{Bun}_{2}(C)_{\mathcal{O}\left(\sum_{i} P_{i}\right)}$, twisted by $\operatorname{det}^{-2}$, where det is the determinant line bundle over $\operatorname{Bun}_{2}(C)_{\mathcal{O}\left(\sum_{i} P_{i}\right)}$ (see [1] and sect. 6).

Proposition 1.2. $\mathcal{H}_{\underline{P}}^{*}(\operatorname{det})$ is isomorphic with $\mathcal{O}(1)^{\bigotimes 3 g}$.

Let $a_{\underline{P}}(\underline{\ell}, z)$ be the element of $\overline{\mathfrak{g}} \otimes \mathbb{C}\left[\ell_{i}, \partial_{\ell_{i}}, i=1, \ldots, 3 g\right]\left[\operatorname{Den}(\underline{P}, \underline{\ell})^{-1}\right] \otimes \Omega(C)$ equal to

$$
\begin{aligned}
& a_{\underline{P}}(\underline{\ell}, z)=-\frac{1}{\operatorname{Den}(\underline{P}, \underline{\ell})} \sum_{i} G\left(P_{i}, z\right) d P_{i}\left\{\left(\frac{1}{2} h+\ell_{i} f\right) \operatorname{Den}(\underline{P}, \underline{\ell})_{\mid \ell_{i}=0}\right. \\
& \left.-\left(e+\ell_{i}^{2} f\right) \partial_{\ell_{i}} \operatorname{Den}(\underline{P}, \underline{\ell})_{\mid \ell_{i}=0}+\left(2 \ell_{i} e-\ell_{i}^{2} h\right) \partial_{\ell_{i}}^{2} \operatorname{Den}(\underline{P}, \underline{\ell})_{\mid \ell_{i}=0}\right\}_{P_{i}=z},
\end{aligned}
$$


and let $s_{\underline{P}}(\underline{\ell}, z)$ be the element of $\mathbb{C}\left[\ell_{i}, \partial_{\ell_{i}}, i=1, \ldots, 3 g\right]\left[\operatorname{Den}(\underline{P}, \underline{\ell})^{-1}\right] \otimes \Omega^{2}(C)$ $\left(\Omega^{2}(C)\right.$ is the space of rational sections of $\left.\Omega_{C}^{\otimes 2}\right)$ given by

$$
\begin{aligned}
s_{\underline{P}}(\underline{\ell}, z)= & \frac{k}{\operatorname{Den}(\underline{P}, \underline{\ell})} \sum_{i=1}^{3 g} d_{z}\left[G\left(P_{i}, z\right) d P_{i}\right] \\
& \left\{-\frac{1}{2} \operatorname{Den}(\underline{P}, \underline{\ell})_{\mid \ell_{i}=0}+\ell_{i} \partial_{\ell_{i}} \operatorname{Den}(\underline{P}, \underline{\ell})_{\mid \ell_{i}=0}-\ell_{i}^{2} \partial_{\ell_{i}}^{2} \operatorname{Den}(\underline{P}, \underline{\ell})_{\mid \ell_{i}=0}\right\}_{P_{i}=z} .
\end{aligned}
$$

Let us also set $G(z, w) d z=\omega^{(z)}(w)$,

$$
\begin{aligned}
& \mu_{i, \underline{P}}(\underline{\ell}, z)=\left(e-\ell_{i} h-\ell_{i}^{2} f\right) G\left(z, P_{i}\right) d z+\frac{1}{\operatorname{Den}(\underline{P}, \underline{\ell})} \cdot \\
& \cdot \sum_{j \neq i} \ell_{i j}^{2}\left[\partial_{\ell_{j}}^{2} \operatorname{Den}(\underline{P}, \underline{\ell})_{\mid \ell_{j}=0} e-\frac{1}{2} \partial_{\ell_{j}} \operatorname{Den}(\underline{P}, \underline{\ell})_{\mid \ell_{j}=0} h-\frac{1}{2} \operatorname{Den}(\underline{P}, \underline{\ell})_{\mid \ell_{j}=0} f\right]_{P_{j}=z} G\left(P_{j}, P_{i}\right) d P_{j} \\
& \nu_{\underline{P}}(\underline{\ell}, z)=-\sum_{i}\left(\frac{1}{2} h+\ell_{i} f\right) G\left(z, P_{i}\right) d z+\frac{1}{\operatorname{Den}(\underline{P}, \underline{\ell})} \sum_{i, j ; i \neq j} G\left(P_{j}, P_{i}\right) d P_{j} \ell_{j i} \cdot \\
& \cdot\left[-\partial_{\ell_{j}}^{2} \operatorname{Den}(\underline{P}, \underline{\ell})_{\mid \ell_{j}=0} e+\frac{1}{2} \partial_{\ell_{j}} \operatorname{Den}(\underline{P}, \underline{\ell})_{\mid \ell_{j}=0} h+\frac{1}{2} \operatorname{Den}(\underline{P}, \underline{\ell})_{\mid \ell_{j}=0} f\right]_{P_{j}=z}
\end{aligned}
$$

and

$$
\ell_{\underline{P}}^{\text {diff }}(z):=\sum_{i} \mu_{i, \underline{P}}(\underline{\ell}, z) \partial_{\ell_{i}}-k \nu_{\underline{P}}(\underline{\ell}, z)
$$

$\ell_{\underline{P}}^{\text {diff }}(z)$ is an element of $\overline{\mathfrak{g}} \otimes \mathbb{C}\left[\ell_{i}, \partial_{\ell_{i}}, i=1, \ldots, 3 g\right]\left[\operatorname{Den}(\underline{P}, \underline{\ell})^{-1}\right] \otimes \Omega(C)$.

Let us set $\sum_{\alpha} x_{\alpha} \otimes y_{\alpha}=e \otimes f+f \otimes e+\frac{1}{2} h \otimes h$ and

$$
T_{\underline{P}}^{\text {diff }}(z)=\sum_{\alpha} \ell_{\underline{P}, x_{\alpha}}^{\text {diff }}(z) \ell_{\underline{P}, y_{\alpha}}^{\text {diff }}(z)+\sum_{\alpha} a_{\underline{P}, x_{\alpha}}(\underline{\ell}, z) \ell_{\underline{P}, y_{\alpha}}^{\text {diff }}(z)+s_{\underline{P}}(\underline{\ell}, z) .
$$

Theorem 1.2. Assume that $k+2=0$. Then $T^{\text {diff }}(z)$ is a regular quadratic differential on $C$. Let us $\left(\omega_{\alpha}^{(2)}(z)\right)_{\alpha=1, \ldots, 3 g-3}$ be a basis of $H^{0}\left(C, \Omega_{C}^{2}\right)$ and set

$$
T_{\underline{P}}^{\text {diff }}(z)=\sum_{\alpha=1}^{3 g-3} T_{\underline{P}, \alpha}^{\text {diff }} \omega_{\alpha}^{(2)}(z)
$$

The $T_{\underline{P}, \alpha}^{\text {diff }}$ form a commuting family of differential operators.

For $\sigma$ a section of $\operatorname{det}^{-2}$ on an open subset of the stable locus, we have

$$
T_{\underline{P}, \alpha}^{\text {diff }}\left(\mathcal{H}_{\underline{P}}^{*} \sigma\right)=\mathcal{H}_{\underline{P}}^{*}\left[T_{\alpha}^{B D}(\sigma)\right]
$$


1.2.4. Functional parametrization of conformal blocks. Let us denote by $A: C \rightarrow$ $J^{1}(C)$ the Abel-Jacobi map of $C$ (see sect.2.3.1). Assume that we have $\sum_{i} A\left(P_{i}\right)=$ $3 g A\left(P_{0}\right) . \operatorname{Bun}_{2}(C)_{\mathcal{O}\left(\sum_{i} P_{i}\right)}$ is then canonically isomorphic to $\operatorname{Bun}_{2}(C)_{\mathcal{O}\left(3 g P_{0}\right)}$. Let $z_{P_{0}}$ be a local coordinate at $P_{0}$, let $\mathcal{K}=\mathbb{C}\left(\left(z_{P_{0}}\right)\right)$ and $\mathcal{O}=\mathbb{C}\left[\left[z_{P_{0}}\right]\right]$. Then we construct an element

$$
g_{\underline{P}, \underline{\ell}} \in G L_{2}(\mathcal{K})
$$

such that its class in the double quotient $G L_{2}\left(H^{0}\left(C \backslash\left\{P_{0}\right\}, \mathcal{O}_{C}\right)\right) \backslash G L_{2}(\mathcal{K}) / G L_{2}(\mathcal{O})=$ $\coprod_{l \in \mathbb{Z}} \operatorname{Bun}_{2}(C)_{\mathcal{O}\left(l P_{0}\right)}$ corresponds to that of $\mathcal{H}_{\underline{P}}(\underline{\ell})$ (Lemma 4.1).

Let us set $G=S L_{2}$. Let $\widetilde{T}_{G(\mathcal{K})}$ be the automorphism of $G(\mathcal{K})$ defined as conjugation by $\left(\begin{array}{cc}z_{P_{0}} & 0 \\ 0 & 1\end{array}\right)$. Let $G(\mathcal{K}) \rtimes \mathbb{Z}$ be the semidirect product of $G(\mathcal{K})$ by this automorphism. Then we have a sequence of group inclusions

$$
G(\mathcal{K}) \subset G(\mathcal{K}) \rtimes \mathbb{Z} \subset G L_{2}(\mathcal{K}),
$$

and the analogous sequence for centrally extended groups $\widehat{G(\mathcal{K})} \subset \widehat{G(\mathcal{K})} \rtimes \mathbb{Z} \subset$ $\left.\widehat{G L_{2}(\mathcal{K}}\right)$, (sect. 4.1.2). (The advantage of working with $G(\mathcal{K}) \rtimes \mathbb{Z}$ rather than $G L_{2}(\mathcal{K})$ is that is does not require the introduction of an additional Heisenberg algebra.) Moreover, $G L_{2}(\mathcal{K})$ is the product of $G(\mathcal{K}) \rtimes \mathbb{Z}$ by its diagonal subgroup. We construct then an element $\widetilde{g}_{\underline{\ell}, \underline{P}}$ of $G(\mathcal{K}) \rtimes \mathbb{Z}$, which is the product of $g_{\underline{\ell}, \underline{P}}$ by a diagonal element of $G L_{2}(\mathcal{K})$.

In sect. 4.2, we also classify the integrable irreducible representations of $G(\mathcal{K}) \rtimes$ $\mathbb{Z}$. Let $\mathbb{W}$ be such a representation. $\mathbb{W}$ can be constructed as the quotient of a module induced by a $\overline{\mathfrak{g}}$-module $W$; we have then an inclusion $W \subset \mathbb{W}$. The space of conformal blocks of $\mathbb{W}$ is then the space $C B(\mathbb{W})$ of linear forms on $\mathbb{W}$, invariant by $\overline{\mathfrak{g}} \otimes H^{0}\left(C \backslash\left\{P_{0}\right\}, \mathcal{O}_{C}\right)$.

Let $\psi$ be an element of $C B(\mathbb{W})$. We associate to it the quantity

$$
f_{\psi}(\underline{\ell}, \underline{P} \mid v)=\left\langle\psi, \widetilde{g}_{\underline{\ell}, \underline{P}} v\right\rangle,
$$

which we view as a function from $\left(\mathbb{C} P^{1}\right)^{3 g}$ to $W^{*}$.

Proposition 1.3. (functional properties of $f_{\psi}(\underline{\ell}, \underline{P} \mid v)$ ) For any $\underline{P}$, the map $v \mapsto$ $f_{\psi}(\underline{\ell}, \underline{P} \mid v)$ belongs to $\operatorname{Hom}_{\overline{\mathfrak{g}}}\left(W, \mathbb{C}\left[\ell_{1}\right]_{\leq k} \otimes \cdots \otimes \mathbb{C}\left[\ell_{3 g}\right]_{\leq k}\right)$.

Here $\mathbb{C}[\ell]_{\leq k}$ is the space of polynomials in $\ell$ of degree $\leq k$; it is endowed with its standard $\overline{\mathfrak{g}}$-module structure.

As we will see, the map $\psi \mapsto f_{\psi}$ should be considered as a "quantization" of the cotangent to the Hecke map $T^{*} \mathcal{H}_{\underline{P}}$.

The variation of $f_{\psi}(\underline{\ell}, \underline{P} \mid v)$ with $\underline{P}$ is described as follows:

Proposition 1.4. Let $\widetilde{f}_{\psi}(\underline{P}, \underline{\ell} \mid v)$ be a function on $C^{3 g} \times\left(\mathbb{C} P^{1}\right)^{3 g}$ with values in $W^{*}$, whose restriction to $\left(C^{3 g}\right)_{P_{0}} \times\left(\mathbb{C} P^{1}\right)^{3 g}$ coincides with $f_{\psi}(\underline{P}, \underline{\ell} \mid v)$. We have

$$
\partial_{P_{i}} \widetilde{f}_{\psi}(\underline{\ell}, \underline{P} \mid v)=\left(\Lambda_{i} \tilde{f}_{\psi}\right)(\underline{\ell}, \underline{P} \mid v)+\sum_{a} \omega_{a}\left(P_{i}\right)\left(\mathcal{A}_{a} \tilde{f}_{\psi}\right)(\underline{\ell}, \underline{P} \mid v)
$$


where

$$
\begin{aligned}
& \Lambda_{i}=\sum_{j: j \neq i} r^{\left(P_{i}\right)}\left(P_{j}\right) \ell_{j i} \frac{\ell_{j}}{\ell_{i}}\left(\frac{\partial}{\partial \ell_{j}}-\frac{k}{\ell_{j}}\right) \\
& -\frac{1}{\operatorname{Den}(\underline{P}, \underline{\ell})} \sum_{j, l: j \neq l} r^{\left(P_{l}\right)}\left(P_{j}\right) \ell_{j l}^{2} \frac{1}{\ell_{i}} \operatorname{Den}(\underline{P}, \underline{\ell})_{\mid \ell_{l}=0, P_{l} \rightarrow P_{i}}\left(\frac{\partial}{\partial \ell_{j}}-\frac{k}{\ell_{j}}\right) \\
& -k \frac{1}{\operatorname{Den}(\underline{P}, \underline{\ell})} \sum_{j, l: l \neq j} \frac{\ell_{j}^{2}}{\ell_{i} \ell_{l}} r^{\left(P_{j}\right)}\left(P_{l}\right) \operatorname{Den}(\underline{P}, \underline{\ell})_{\mid \ell_{j}=0, P_{j} \rightarrow P_{i}} \\
& +k\left(\frac{1}{\ell_{i}} e-\frac{1}{2} h\right)+\frac{k}{\operatorname{Den}(\underline{P}, \underline{\ell})} \sum_{j}-\frac{\ell_{j}}{\ell_{i}} \operatorname{Den}(\underline{P}, \underline{\ell})_{\mid \ell_{j}=0, P_{i} \rightarrow P_{j}[}\left[\frac{1}{\ell_{j}} e-h-\ell_{j} f\right],
\end{aligned}
$$

where we denote by $x \in \overline{\mathfrak{g}}$ the operator $f(\underline{P}, \underline{\ell} \mid v) \mapsto f\left(\underline{P}, \underline{\ell} \mid \rho_{W}(x) v\right)$, and $\mathcal{A}_{a}$ are some linear operators.

Here the term $\sum_{a} \omega_{a}\left(P_{i}\right)\left(\mathcal{A}_{a} \tilde{f}_{\psi}\right)(\underline{\ell}, \underline{P} \mid v)$ is the Lagrange multiplier corresponding to the relation $\delta\left(\sum A\left(P_{i}\right)\right)=0$.

1.2.5. Explicit form of the $K Z B$ connection. Let us denote by $\mathcal{M}_{g, 1^{\infty}}$ the moduli space of triples $m_{\infty}=\left(C, P_{0}, t\right)$ of a smooth curve $C$ of genus $g$, a marked point $P_{0}$ of $C$ and a formal coordinate $t$ at $P_{0}$.

$\mathcal{M}_{g, 1 \infty}$ may be identified with the set of all inclusions $R \subset \mathbb{C}((t))$, where $R$ is the coordinate ring of a smooth curve of genus $g$ minus a point.

Let us set $\mathfrak{g}=\overline{\mathfrak{g}} \otimes \mathbb{C}((t)) \oplus \mathbb{C} K$. Consider $\mathbb{W}$ as a $\mathfrak{g}$-module and $\mathfrak{g}^{\text {out,ext }}$ as a Lie subalgebra of $\mathfrak{g}$.

The bundle of conformal blocks on $\mathcal{M}_{g, 1^{\infty}}$ is defined as the subbundle of the constant bundle over $\mathcal{M}_{g, 1^{\infty}}$ with fiber $\mathbb{W}^{*}$, consisting of the $\mathfrak{g}^{\text {out,ext}}$-invariant forms. We denote this bundle by $C B(\mathbb{W})$.

A flat connection is defined on $C B(\mathbb{W})$ as follows (see [21, 4, 6]). Recall first that the map

$$
\mathbb{C}((t)) \frac{\partial}{\partial t} \rightarrow \operatorname{Vect}\left(\mathcal{M}_{g, 1 \infty}\right), \quad \xi \mapsto[\xi],
$$

defined by $\exp (\epsilon[\xi])([R \subset \mathbb{C}((t))])=[(1+\epsilon \xi)(R) \subset \mathbb{C}((t))]$, when $\epsilon^{2}=0$, induces a Lie algebra morphism from vector fields on the formal disc to vector fields on $\mathcal{M}_{g, 1^{\infty}}$. For $m_{\infty} \mapsto \psi\left(m_{\infty}\right)$ a local section of $C B(\mathbb{W})$, let us set

$$
\nabla_{[\xi]}^{C B} \psi\left(m_{\infty}\right)=\partial_{[\xi]} \psi\left(m_{\infty}\right)-\psi\left(m_{\infty}\right) \circ T[\xi],
$$

where $T[\xi]=\operatorname{res}_{z=P_{0}}(\xi(z) T(z))$.

Let us denote by $\mathcal{F}$ the bundle over $\mathcal{M}_{g, 1^{\infty}}$, whose fiber $\mathcal{F}\left(m_{\infty}\right)$ at $m_{\infty}$ is the space of functions $f(\underline{P}, \underline{\ell} \mid v)$ from $\left(C^{3 g}\right)_{P_{0}} \times\left(\mathbb{C} P^{1}\right)^{3 g}$ to $W^{*}$, such that

$$
\partial_{P_{i}} f(\underline{P}, \underline{\ell} \mid v)=\Lambda_{i} f(\underline{P}, \underline{\ell} \mid v)+\sum_{a} \omega_{a}\left(P_{i}\right) f_{a}(\underline{P}, \underline{\ell} \mid v),
$$

where $f_{a}(\underline{P}, \underline{\ell} \mid v)$ are functions from $\left(C^{3 g}\right)_{P_{0}} \times\left(\mathbb{C} P^{1}\right)^{3 g}$ to $W^{*}$. 
HECKE-TYURIN PARAMETRIZATION OF THE HITCHIN AND KZB SYSTEMS

Define $\mathcal{M}_{g, 1^{\infty},(3 g)}$ as the moduli space of all pairs $\left(m_{\infty}, \underline{P}\right)$, such that $\sum_{i} A\left(P_{i}\right)=$ $3 g A\left(P_{0}\right)$. Let $\xi$ be a formal vector field $\xi \in \mathbb{C}((t)) \frac{\partial}{\partial t}$, such that

$$
\operatorname{res}_{P_{0}}\left(\xi \omega_{a} \frac{d b_{\underline{P}}}{b_{\underline{P}}}\right)=0
$$

for each $a=1, \ldots, g$. Then $\xi$ induces a vector field $[\xi]$ at $\left(m_{\infty}, \underline{P}\right)$, defined by $(1+\epsilon[\xi])\left(m_{\infty}, \underline{P}\right)=\left(m_{\infty}^{\prime}, \underline{P}^{\prime}\right)$, where $m_{\infty}^{\prime}$ in the inclusion $(1+\epsilon \xi)(R) \subset \mathbb{C}((t))$, and $P_{i}^{\prime}$ correspond the characters $\chi_{P_{i}} \circ(1-\epsilon \xi)$ of $R$, where $\chi_{P_{i}}$ is the character of $R$ corresponding to $P_{i}$ (see [6], Lemma 5.1).

Proposition 1.5. The rule

$$
\left(\nabla_{[\xi]}^{\mathcal{F}} f\right)\left(m_{\infty}, \underline{P}, \underline{\ell} \mid v\right)=\partial_{[\xi]} f\left(m_{\infty}, \underline{P}, \underline{\ell} \mid v\right)-\operatorname{res}_{z=P_{0}}\left(\xi(z) T^{\text {diff }}(z) f\left(m_{\infty}, \underline{P}, \underline{\ell} \mid v\right)\right)
$$

where $\left.f\left(m_{\infty}, \underline{P}, \underline{\ell} \mid v\right)\right)$ is a local section of $\mathcal{F}$, defines a flat connection $\nabla^{\mathcal{F}}$ on $\mathcal{F}$.

Define corr as the bundle morphism from $C B(\mathbb{W})$ to $\mathcal{F}$, such that $\operatorname{corr}(\psi)\left(m_{\infty}, \underline{P}, \underline{\ell} \mid v\right)=$ $\left\langle\psi, \rho_{\mathbb{W}}^{\text {group }}\left(\widetilde{g}_{\underline{P}, \underline{\ell}}\right)(v)\right\rangle$. Then $\nabla^{\mathcal{F}} \circ \operatorname{corr}=\operatorname{corr} \circ \nabla^{C B}$.

We end the paper with the construction of a filtration naturally attached to $\mathcal{H}_{\underline{P}}$ (sect. 8), and questions about its possible relation with formulas of [9, 18].

1.3. Generalization to arbitrary semisimple groups. The above parametrization maps are generalized as follows. Let $G$ be a reductive complex group. Let $\mathcal{O}$ be a formal series ring $\mathbb{C}[[t]]$, and let $\mathcal{K}$ be its fraction field $\mathbb{C}((t))$. Let Grass be the affine Grassmann variety Grass $=G(\mathcal{K}) / G(\mathcal{O})$. Grass is the union of all $\operatorname{Grass}_{\chi}=G(\mathcal{O}) w_{\chi} G(\mathcal{O}) / G(\mathcal{O})$, where $\chi$ belongs to the semigroup $P_{+}$of dominant coweights of the Langlands dual group ${ }^{L} G$ of $G$, and $w_{\chi}$ is associated translation of the affine Weyl group of $G$. Let $\underline{\chi}=\left(\chi_{i}\right)_{i=1, \ldots, N}$ be a family of elements of $P_{+}$. We have then Hecke maps

$$
\mathcal{H}_{\underline{P}, \underline{\chi}}: \prod_{i=1}^{N} \operatorname{Grass}_{\chi_{i}} \rightarrow \operatorname{Bun}_{G}(C),
$$

obtained from the natural map $G^{\text {out }, \underline{P}} \backslash \prod_{i=1}^{N} \operatorname{Grass}_{\chi_{i}} \rightarrow \operatorname{Bun}_{G}(C)$, where $G^{\text {out }, \underline{P}}$ is the group of regular maps from $C \backslash\left\{P_{i}, i=1, \ldots, N\right\}$ to $G$.

Moreover, the map [class of $\left.g w_{\chi} g^{\prime}\right] \mapsto$ class of $g$ ] defines isomorphisms

$$
\operatorname{Grass}_{\chi} \rightarrow G(\mathcal{O}) /\left[{ }^{w_{\chi}} G(\mathcal{O}) \cap G(\mathcal{O})\right]=N(\mathcal{O}) /\left[{ }^{w_{\chi}} N(\mathcal{O}) \cap N(\mathcal{O})\right],
$$

where $N$ is the positive unipotent subgroup of $G$, and we set ${ }^{g} h=g h g^{-1}$.

1.4. Comparison with earlier work. In [5], G. Felder and one of us introduced another parametrization of the KZB connection. Let us explain its relation to the approch of the present work. The work [5] was based on a variant of the Feigin-Stoyanovski description of the conformal blocks, which relies on the map

$$
F S: \operatorname{Bun}_{B}(C)_{P_{0}} \rightarrow \operatorname{Bun}_{G}(C) .
$$


Here $P_{0}$ is a fixed point of $C$ and $\operatorname{Bun}_{B}(C)_{P_{0}}$ is the union of all $\operatorname{Bun}_{B}(C)_{P_{0}, \chi}$, which is the moduli space of all $B$-bundles over $C$, with associated $T$-bundle isomorphic to $\oplus_{i}$ simple $\mathcal{O}\left(\left\langle\chi, \alpha_{i}\right\rangle P_{0}\right)$. Here $B$ the Borel subgroup of $G$ containing $N$, and $T$ is the associated Cartan subgroup. Let us denote by $F S_{\chi}$ the restriction of $F S$ to $\operatorname{Bun}_{B}(C)_{P_{0}, \chi}$.

The Hecke map $\mathcal{H}_{P_{0}, \chi}$ factors through the above map $F S_{\chi}$. Indeed, the composition of $\mathcal{H}_{P_{0}, \chi}$ with the isomorphism (3) is the map

$$
N(\mathcal{O}) /\left[{ }^{w_{\chi}} N(\mathcal{O}) \cap N(\mathcal{O})\right] \rightarrow \operatorname{Bun}_{G}(C),
$$

class of $n \mapsto$ class of $n w_{\chi}$. On the other hand, $\operatorname{Bun}_{B}(C)_{P_{0}, \chi}$ is equal to $N(R) \backslash$ $\left\{N(\mathcal{K}) w_{\chi}\right\} / N(\mathcal{O})$, where $R=H^{0}\left(C \backslash\left\{P_{0}\right\}, \mathcal{O}_{C}\right)$. The map (四) therefore factors through $\operatorname{Bun}_{B}(C)_{P_{0}, \chi}$.

It is probable that the maps $\mathcal{H}_{\underline{P}, \chi}$ have the following behavior. When $P_{i}$ and $P_{j}$ tend to some point $P$, the limit of $\mathcal{H}_{\underline{P}, \underline{\chi}}$ should be related to $\mathcal{H}_{\underline{P}^{\prime}, \underline{\chi}^{\prime}}$, where $\underline{P}^{\prime}=\left(\left(P_{\alpha}\right)_{\alpha \neq i, j}, P\right)$ and $\underline{\chi}^{\prime}=\left(\left(\chi_{\alpha}\right)_{\alpha \neq i, j}, \chi_{i}+\chi_{j}\right)$. One could then think that the maps $F S$, which involve all coweights of ${ }^{L} G$, can be obtained as limits of the maps $\mathcal{H}_{\underline{P}, \underline{\chi}}$, where $\chi_{i}$ are simple coweights of ${ }^{L} G$, and that one can obtain the differential systems expressed in [5] from those of the present work by a similar limiting procedure.

1.5. Open problems. 1) It is easy to write variants of our parametrization of the Beilinson-Drinfeld $\mathcal{D}$-modules, corresponding to the parametrization $\left(\mathbb{C} P^{1}\right)^{3 g-3} \rightarrow$

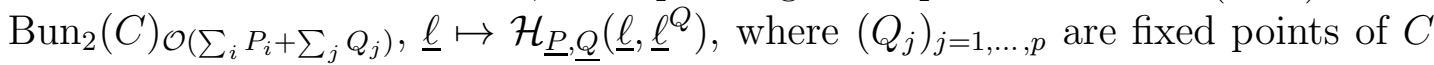
and $\underline{\ell}^{Q}$ a fixed element of $\left(\mathbb{C} P^{1}\right)^{p}$. Using these variants, one could hope to check explicitly the Hecke eigenvalue property of the Beilinson-Drinfeld $\mathcal{D}$-modules.

2) It should also be possible to write the analogues of our systems in the $\mathfrak{s l}_{n}$ case; it that case the parametrization maps could be $\left(\mathbb{C} P^{n}\right)^{n+1} \rightarrow \operatorname{Bun}_{n}(C)_{\mathcal{L}}$.

3) In [5], II, we introduced commuting difference analogues of the differential operators provided by action of the Sugawara tensor at critical level. It would be interesting to find commuting difference analogues of the operators $T^{\operatorname{diff}}(z)$ of the present work.

4) In sect. 8, we introduce filtrations of the conformal blocks, provided by the maps $\mathcal{H}_{\underline{P}}$; it would be interesting to compute the corresponding $q$-dimensions.

5) One could expect simple transformations from our version of the KZB system to the versions of van Geemen-Previato ([12]) and of Gawȩdzki-Tran ([11]), when the genus of $C$ equals 2 .

1.6. The first author would like to express his thanks to G. Felder for many conversations on conformal blocks and collaboration in [5, 6]. We also would like to thank V. Drinfeld, whose talk at the 1994 Alushta conference (Ukraine) introduced us to the subject of this work. We also would like to thank A. Lossev and E. Vasserot for discussions related to this work. 
HECKE-TYURIN PARAMETRIZATION OF THE HITCHIN AND KZB SYSTEMS

We also would like to express our gratitute to A. Alekseev, L. Faddeev and H. Grosse for an invitation at ESI (Vienna) in August 1999, during which this work was started. The second author was partially supported by grant RFFI-98-0100327.

\section{Hecke parametrization of $\operatorname{Bun}_{2}(C)_{\mathcal{O}\left(\sum_{i} P_{i}\right)}$}

Let $C$ be a smooth, complete, connected complex curve, let $g$ be its genus. Let us set $G=S L_{2}$, so $G(\mathbb{C})=S L_{2}(\mathbb{C})$. For $\mathcal{L}$ a line bundle over $C$, let us denote by $\operatorname{Bun}_{2}(C)_{\mathcal{L}}$ the moduli space of rank 2 vector bundles over $C$, whose determinant bundle is isomorphic to $\mathcal{L}$.

2.1. Theta-functions. Let us fix our conventions for theta-functions. We set, for $\lambda$ in $\mathbb{C}^{g}$, and $\tau=\left(\tau_{a b}\right)_{a, b=1, \ldots, g}$ a symmetric matrix with negative real part,

$$
\Theta(\lambda \mid \tau)=\sum_{m \in \mathbb{Z}^{g}} \exp \left(\frac{1}{2} m \tau m^{t}+m \lambda^{t}\right) .
$$

We set $\tau(C)_{a b}=\int_{B_{a}} \omega_{b}$, and $L(C)=(2 i \pi \mathbb{Z})^{g}+\tau \mathbb{Z}^{g}$. Then the degree zero part $J^{0}(C)$ of the Jacobian of $C$ is isomorphic to $\mathbb{C}^{g} / L(C)$. The Abel-Jacobi map sends a divisor $\sum_{i} n_{i} P_{i}$ of $C$ of total degree zero to the class of the vector $\left(\sum_{i} \int_{x_{0}}^{P_{i}} \omega_{a}\right)_{a=1, \ldots, g}$ (which is independent of the choice of $x_{0}$ and of the integration contours).

The vector of Riemann constants $\Delta$ is the element of the degree $g-1$ part $J^{g-1}(C)$ of the Jacobian of $C$ such that the identity $\Theta\left(\sum_{i=1}^{g-1} x_{i}-\Delta\right)=0$. We also fix an odd theta-cahracteristic $\delta$ (the product of $\lambda \mapsto \Theta(\lambda+\delta-\Delta)$ by an exponential factors is then an odd function of $\lambda$ ).

Let us set

$$
r^{(P)}(z)=d_{P} \ln \Theta(A(P)-A(z)+\delta-\Delta) .
$$

Then we have

$$
r^{(P)}\left(\gamma_{A_{a}} z\right)=r^{(P)}(z), \quad r^{(P)}\left(\gamma_{B_{a}} z\right)=r^{(P)}(z)+\omega_{a}(P) .
$$

2.2. The Hecke parametrization map $\mathcal{H}_{\underline{P}}$. Let $\mathcal{E}$ be a vector bundle of rank 2 over $C$. Let $\underline{P}=\left(P_{i}\right)_{i=1, \ldots, N}$ be a family of points on $C$. For $P$ a point of $C$, let us denote by $\mathcal{E}_{P}$ the fiber of $\mathcal{E}$ at $P$. Let $\underline{\ell}=\left(\ell_{i}\right)_{i=1, \ldots, N}$ be an element of $\prod_{i=1}^{N} \mathbb{P}\left(\mathcal{E}_{P_{i}}\right)$. The Hecke modification $\mathcal{H}_{\underline{P}, \underline{\ell}}(\mathcal{E})$ of $\mathcal{E}$ along $(\underline{P}, \underline{\ell})=\left(P_{i}, \ell_{i}\right)_{i=1, \ldots, N}$ is defined as the sheaf whose space of sections over an open subset $U$ of $C$ is $\{\sigma$ : rational section of $\mathcal{E}$ over $U$, regular outside $\left\{P_{i}, i=1, \ldots, N\right\}$, with a simple pole at each $P_{i}$ and residue in the line $\ell_{i}$.

Define a map $\mathcal{H}_{\underline{P}}:\left(\mathbb{C} P^{1}\right)^{N} \rightarrow \operatorname{Bun}_{2}(C)_{\mathcal{O}\left(\sum_{i} P_{i}\right)}$ by

$$
\mathcal{H}_{\underline{P}}(\underline{\ell}):=\mathcal{H}_{\underline{P}, \underline{\ell}}\left(\mathcal{O}_{C}^{2}\right)
$$


where $\underline{\ell}=\left(\ell_{1}, \ldots, \ell_{N}\right)$ belongs to $\left(\mathbb{C} P^{1}\right)^{N}$ and $\mathcal{O}_{C}^{2}$ denotes the trivial bundle of rank 2 over $C$. Since the group of automorphisms of $\mathcal{O}_{C}^{2}$ is isomorphic to $G L_{2}(\mathbb{C})$, $\mathcal{H}_{\underline{P}}$ factors through $\left(\mathbb{C} P^{1}\right)^{N} \rightarrow\left(\mathbb{C} P^{1}\right)^{N} / G L_{2}(\mathbb{C})=\left(\mathbb{C} P^{1}\right)^{N} / G(\mathbb{C})$.

\subsection{Properties of $\mathcal{H}_{\underline{P}}$ in the case $N=3 g$ (proof of Thm. 1.1).}

2.3.1. The preimage of stable bundles (proof of the first part of Thm. 1.1). In this section, we will characterize the preimage by the map $\mathcal{H}_{\underline{P}}$ of the subset of $\operatorname{Bun}_{2}(C)_{\mathcal{O}\left(\sum_{i} P_{i}\right)}$ consisting of stable bundles.

Let us define $M(\underline{P}, \underline{\ell})$ as the $3 g \times 3 g$ matrix with matrix elements

$$
M(\underline{P}, \underline{\ell})_{i, 3 j+\alpha}=\omega_{a}\left(P_{i}\right) \ell_{i}^{\alpha} .
$$

for $i=1, \ldots, 3 g, a=1, \ldots, g, \alpha=1,2,3$.

Lemma 2.1. We have

$$
\operatorname{det} M(\underline{P}, \underline{\ell})=\operatorname{Den}(\underline{P}, \underline{\ell}),
$$

where $\operatorname{Den}(\underline{P}, \underline{\ell})$ is defined by (1).

Recall that the bundle $\mathcal{E}$ is called stable iff $H^{0}(C, \operatorname{End}(\mathcal{E}))=\mathbb{C}$. The set of stable bundles has the structure of a quasi-projective variety ([20]).

Proposition 2.1. Assume that all $\ell_{i}$ are $\neq \infty$. Then if $\operatorname{Den}(\underline{P}, \underline{\ell}) \neq 0, \mathcal{H}_{\underline{P}}(\underline{\ell})$ is stable.

Proof. Let us set $\mathcal{E}=\mathcal{H}_{P}(\underline{\ell})$. Let $\operatorname{End}(\mathcal{E})_{0}$ be the subbundle of $\operatorname{End}(\mathcal{E})$ formed of traceless endomorphisms. We derive from the exact sequence of sheaves

$$
0 \rightarrow \operatorname{End}(\mathcal{E})_{0} \rightarrow \operatorname{End}(\mathcal{E}) \rightarrow \mathcal{O}_{C} \rightarrow 0,
$$

the exact sequence $0 \rightarrow H^{0}\left(C, \operatorname{End}(\mathcal{E})_{0}\right) \rightarrow H^{0}(C, \operatorname{End}(\mathcal{E})) \rightarrow \mathbb{C}$. The stability condition is therefore equivalent to $H^{0}\left(C, \operatorname{End}(\mathcal{E})_{0}\right)=0$.

Let us denote by $\mathbb{C}(C)$ the function field of $C . H^{0}\left(C, \operatorname{End}(\mathcal{E})_{0}\right)$ is isomorphic to the subspace of $\overline{\mathfrak{g}} \otimes \mathbb{C}(C)$ formed of the rational functions $\varphi: C \rightarrow \overline{\mathfrak{g}}$,

i) regular outside $\left\{P_{i}\right\}$,

ii) which have simple poles at each $P_{i}$ and residue at that point in $\mathbb{C}(-e+$ $\left.\ell_{i} h+\ell_{i}^{2} f\right)$,

iii) such that if we write $\varphi=\varphi_{e} e+\varphi_{h} h+\varphi_{f} f$, with $\varphi_{e}, \varphi_{h}, \varphi_{f}$ in $\mathbb{C}(C)$, $-\ell_{i}^{2} \varphi_{e}-2 \ell_{i} \varphi_{h}+\varphi_{f}$ is regular at $P_{i}$.

Conditions i) and ii) mean that for some constants $\left(\lambda_{i}\right)_{i=1, \ldots, 3 g}$ and $C_{e}, C_{h}, C_{f}$, we have

$$
\varphi=\sum_{i=1}^{3 g} \lambda_{i}\left(-e+\ell_{i} h+\ell_{i}^{2} f\right) r^{\left(P_{i}\right)}(z)+C_{e} e+C_{h} h+C_{f} f
$$

and

$$
\sum_{i} \lambda_{i} \ell_{i}^{\alpha} \omega_{a}\left(P_{i}\right)=0, \quad a=1, \ldots, g, \quad \alpha=0,1,2,
$$


and condition iii) is equivalent to

$$
\sum_{j \neq i} \lambda_{j} \ell_{i j}^{2} r^{\left(P_{j}\right)}\left(P_{i}\right)+C_{f}-2 \ell_{i} C_{h}-\ell_{i}^{2} C_{e}=0 \text { for each } i=1, \ldots, 3 g .
$$

Since $\operatorname{Den}(\underline{P}, \underline{\ell}) \neq 0,(6)$ implies that all $\lambda_{i}$ are zero. Moreover, $\operatorname{Den}(\underline{P}, \underline{\ell}) \neq 0$ also implies that $\operatorname{card}\left\{\ell_{i} ; i=1, \ldots, 3 g\right\}$ is $\geq 3$. Then (7) implies that $C_{f}=C_{h}=$ $C_{e}=0$.

\subsubsection{The fibers of $\mathcal{H}_{\underline{P}}$. In this section, we prove:}

Proposition 2.2. For any $\underline{\ell}$ such that $\operatorname{Den}(\underline{P}, \underline{\ell}) \neq 0$, the set $\mathcal{H}_{\underline{P}}^{-1}\left(\mathcal{H}_{\underline{P}}(\underline{\ell})\right)$ is finite.

Proof. Let $\underline{\ell}$ and $\underline{\ell}^{\prime}$ belong to $\left(\mathbb{C} P^{1}\right)^{3 g}$. Assume that $\operatorname{Den}(\underline{P}, \underline{\ell}) \neq 0$. The bundles $\mathcal{H}_{\underline{P}}(\underline{\ell})$ and $\mathcal{H}_{\underline{P}}\left(\underline{\ell}^{\prime}\right)$ are isomorphic iff there exists a rational function $M(z)$ defined on $C$, with values in $S L_{2}(\mathbb{C})$, regular except for simple poles at the $P_{i}$, where the residue is proportional to $\left(\begin{array}{c}1 \\ \ell_{i}^{\prime}\end{array}\right)\left(\begin{array}{ll}\ell_{i} & -1\end{array}\right) . M(z)$ is then written

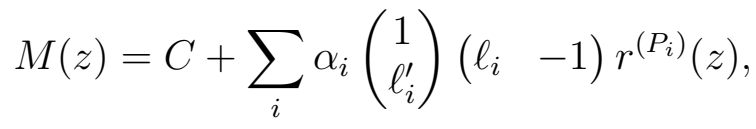

where $C$ is in $M_{2}(\mathbb{C})$ and where $\alpha_{i}$ are tangent vectors at $P_{i}$, satisfying

$$
\sum_{i} \omega_{a}\left(P_{i}\right) \alpha_{i}\left(\begin{array}{l}
1 \\
\ell_{i}^{\prime}
\end{array}\right)\left(\begin{array}{ll}
\ell_{i} & -1
\end{array}\right)=0, \quad a=1, \ldots, g
$$

and

$$
\left(\begin{array}{ll}
\ell_{i}^{\prime} & -1
\end{array}\right) C\left(\begin{array}{l}
1 \\
\ell_{i}
\end{array}\right)+\sum_{j \neq i} r^{\left(P_{j}\right)}\left(P_{i}\right) \alpha_{j}\left(\ell_{i}^{\prime}-\ell_{j}^{\prime}\right)\left(\ell_{j}-\ell_{i}\right)=0, \quad i=1, \ldots, 3 g .
$$

Let us set $\beta_{i}=\alpha_{i} \ell_{i}^{\prime}$. Since $\operatorname{Den}(\underline{P}, \underline{\ell}) \neq 0$, (8) means that the vector $\left(\alpha_{i}, \beta_{i}\right)_{i=1, \ldots, 3 g}$ belongs to a $2 g$-dimensional vector space. On the other hand, (9) is equivalent to

$$
\left(\begin{array}{ll}
\beta_{i} & -\alpha_{i}
\end{array}\right) C\left(\begin{array}{c}
1 \\
\ell_{i}
\end{array}\right)+\sum_{j \neq i} r^{\left(P_{j}\right)}\left(P_{i}\right)\left(\alpha_{j} \beta_{i}-\alpha_{i} \beta_{j}\right)\left(\ell_{j}-\ell_{i}\right)=0, \quad i=1, \ldots, 3 g
$$

which is a system of $3 g$ quadratic conditions on this vector. If these conditions are satisfied, the condition that $\operatorname{det} M(z)=1$ is then equivalent to $\operatorname{det} M\left(P_{0}\right)=$ 1. The set of $\left(C, \alpha_{i}, \beta_{i}\right)$ satisfying these conditions is therefore a subvariety of $M_{2}(\mathbb{C}) \times \mathbb{C}^{3 g} \times \mathbb{C}^{3 g}$, and $\mathcal{H}_{\underline{P}}\left(\mathcal{H}_{\underline{P}}^{-1}(\underline{\ell})\right)$ is the image of this variety by the morphism $\left(C, \alpha_{i}, \beta_{i}\right)_{i=1, \ldots, 3 g} \mapsto\left(\beta_{i} / \alpha_{i}\right)_{i=1, \ldots, 3 g}$.

Let us fix now $\underline{\ell}^{\prime}$ in $\mathcal{H}_{\underline{P}}\left(\mathcal{H}_{\underline{P}}^{-1}(\underline{\ell})\right)$. It follows from Prop. 2.1 that $\operatorname{Den}(\underline{P}, \underline{\ell}) \neq 0$. Let us compute the tangent space of $\mathcal{H}_{\underline{P}}\left(\mathcal{H}_{\underline{P}}^{-1}(\underline{\ell})\right)$ at $\underline{\ell}^{\prime}$. Let $\delta \underline{\ell}^{\prime}$ be an infinitesimal 
element of this vector space. To it is associated a matrix corresponding to the isomorphism from $\mathcal{H}\left(\underline{P}, \underline{\ell}^{\prime}\right)$ to $\mathcal{H}\left(\underline{P}^{\prime}, \underline{\ell}^{\prime}+\delta \underline{\ell}^{\prime}\right)$. We have

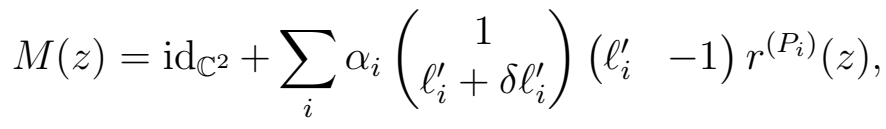

where $\alpha_{i}$ are infinitesimals. They should satisfy the equations

$$
\sum_{i} \alpha_{i} \omega_{a}\left(P_{i}\right)\left(\begin{array}{c}
1 \\
\ell_{i}^{\prime}+\delta \ell_{i}^{\prime}
\end{array}\right)\left(\begin{array}{ll}
\ell_{i}^{\prime} & -1
\end{array}\right)=0
$$

for each $a$, therefore

$$
\sum_{i} \alpha_{i} \omega_{a}\left(P_{i}\right)=\sum_{i} \alpha_{i} \ell_{i}^{\prime} \omega_{a}\left(P_{i}\right)=\sum_{i} \alpha_{i} \ell_{i}^{\prime 2} \omega_{a}\left(P_{i}\right)=0 .
$$

As $\operatorname{Den}\left(\underline{P}, \underline{\ell}^{\prime}\right) \neq 0$, this implies that the $\alpha_{i}$ are all zero.

Remark 1. The subvariety of $M_{2}(\mathbb{C}) \times \mathbb{C}^{3 g} \times \mathbb{C}^{3 g}$ defined by conditions (8) and (9) (without the condition $\operatorname{det} M\left(P_{0}\right)=1$ ) has components of dimension $>0$. This makes it difficult to evaluate the degree of $\mathcal{H}_{\underline{P}}$.

2.3.3. $\mathcal{H}_{\underline{P}}$ is a finite etale morphism (proof of the second part of Thm. 1.1). In [20], $\operatorname{Bun}_{2}(C)_{\mathcal{O}\left(\sum_{i} P_{i}\right)}^{\text {stable }}$ is endowed with the structure of a quasi-projective variety. On the other hand, since the action of $G$ on $\left\{\underline{\ell} \in\left(\mathbb{C} P^{1}\right)^{3 g} \mid \operatorname{Den}(\underline{P}, \underline{\ell}) \neq 0\right\}$ is free, the quotient $\left\{\underline{\ell} \in\left(\mathbb{C} P^{1}\right)^{3 g} \mid \operatorname{Den}(\underline{P}, \underline{\ell}) \neq 0\right\} / G$ also has the structure of a quasi-projective variety. One can check using [20] that the restriction of $\mathcal{H}_{P}$ to $\left\{\underline{\ell} \in\left(\mathbb{C} P^{1}\right)^{3 g} \mid \operatorname{Den}(\underline{P}, \underline{\ell}) \neq 0\right\} / G$ is a morphism of varieties.

Moreover, the dimensions of $\left\{\underline{\ell} \in\left(\mathbb{C} P^{1}\right)^{3 g} \mid \operatorname{Den}(\underline{P}, \underline{\ell}) \neq 0\right\} / G$ and $\operatorname{Bun}_{2}(C)_{\mathcal{O}\left(\sum_{i} P_{i}\right)}^{\text {stable }}$ are equal. It follows from Prop. 2.2 that the kernel of the map induced by $\mathcal{H}_{\underline{P}}$ on tangent spaces is zero. It follows that $\mathcal{H}_{\underline{P}}$ induces an isomorphism between the tangent spaces of $\left\{\underline{\ell} \in\left(\mathbb{C} P^{1}\right)^{3 g} \mid \operatorname{Den}(\underline{P}, \underline{\ell}) \neq 0\right\} / G$ at $G \underline{\ell}$ and of $\operatorname{Bun}_{2}(C)_{\mathcal{O}\left(\sum_{i} P_{i}\right)}^{\text {stable }}$ at $\mathcal{H}_{\underline{P}}(\underline{\ell})$. It follows that the restriction $\mathcal{H}_{\underline{P}}$ to $\left\{\underline{\ell} \in\left(\mathbb{C} P^{1}\right)^{3 g} \mid \operatorname{Den}(\underline{P}, \underline{\ell}) \neq 0\right\} / G$ is etale.

Remark 2. The map $\mathcal{H}_{\underline{P}}$ is never surjective; for example, the $p$ large enough, the bundle $\mathcal{O}\left(p P_{0}\right) \oplus \mathcal{O}\left(\sum_{i} P_{i}-p P_{0}\right)$ is not in the image of $\mathcal{H}_{\underline{P}}$. But one may ask whether $\operatorname{Im} \mathcal{H}_{\underline{P}}$ contains all stable bundles when $N=\operatorname{card}\left\{\bar{P}_{i}\right\}$ is large enough.

\section{The Hitchin system in Hecke coordinates}

3.1. The map induced by $\mathcal{H}_{\underline{P}}$ on cotangent bundles. Let us assume that $N=3 g$. Let $\underline{\ell}=\left(\ell_{i}\right)_{i=1, \ldots, 3 g}$ be such that $\operatorname{Den}(\underline{P}, \underline{\ell}) \neq 0$. It follows from Thm. 1.1 that $\mathcal{H}_{\underline{P}}$ is an etale isomorphism at the neighborhood of the class $[\underline{\ell}]$ of $\underline{\ell}$ in $\left(\mathbb{C} P^{1}\right)^{3 g} / G(\mathbb{C})$.

$\mathcal{H}_{\underline{P}}$ therefore induces an isomorphism of cotangent spaces

$$
T^{*} \mathcal{H}_{\underline{P}}: T_{[\underline{\ell}]}^{*}\left[\left(\mathbb{C} P^{1}\right)^{3 g} / G(\mathbb{C})\right] \rightarrow T_{\mathcal{H}_{\underline{P}}(\underline{\ell})}^{*} \operatorname{Bun}_{2}(C)_{\mathcal{O}\left(\sum_{i=1}^{3 g} P_{i}\right)} .
$$


HECKE-TYURIN PARAMETRIZATION OF THE HITCHIN AND KZB SYSTEMS

3.1.1. For $\ell$ in $\mathbb{C} P^{1}, T_{\ell}^{*} \mathbb{C} P^{1}$ is isomorphic to $\mathbb{C}$. Let us denote by $\overline{\mathfrak{g}}$ the Lie algebra $\mathfrak{s l}_{2}(\mathbb{C})$. Let $e, h, f$ be its Chevalley generators, and let $\langle,\rangle_{\overline{\mathfrak{g}}}$ be the invariant form on $\overline{\mathfrak{g}}$ such that $\langle e, f\rangle_{\overline{\mathfrak{g}}}=\frac{1}{2}\langle h, h\rangle_{\overline{\mathfrak{g}}}=1$. We will identify $\overline{\mathfrak{g}}$ with its dual using $\langle,\rangle_{\overline{\mathfrak{g}}} \cdot T^{*} \mathbb{C} P^{1}$ is a symplectic manifold with a Hamiltonian action of $S L_{2}(\mathbb{C})$, and the corresponding moment map $\mu: T^{*} \mathbb{C} P^{1} \rightarrow \overline{\mathfrak{g}}^{*}$ is given by $(\ell, \lambda) \mapsto \lambda(-e+\ell h+$ $\left.\ell^{2} f\right) ; \mu$ coincides with the Springer desingularization.

$T_{[\underline{\ell}]}^{*}\left[\left(\mathbb{C} P^{1}\right)^{3 g} / G(\mathbb{C})\right]$ is therefore isomorphic to $\left(\mu^{3 g}\right)^{-1}(0) / G(\mathbb{C})$; the preimage of $\mathbb{C}^{3 g}$ by the projection $\mu^{-1}(0) \rightarrow\left(\mathbb{C} P^{1}\right)^{3 g}$ is

$$
\left\{(\underline{\ell}, \underline{\lambda})=\left(\ell_{i}, \lambda_{i}\right)_{i=1, \ldots, 3 g} \mid \sum_{i=1}^{3 g} \lambda_{i}=\sum_{i=1}^{3 g} \lambda_{i} \ell_{i}=\sum_{i=1}^{3 g} \lambda_{i} \ell_{i}^{2}=0\right\}
$$

and the action of $G(\mathbb{C})$ on this space is

$$
\left(\begin{array}{ll}
a & b \\
c & d
\end{array}\right) \cdot\left(\ell_{i}, \lambda_{i}\right)_{i=1, \ldots, 3 g}=\left(\frac{a \ell_{i}+b}{c \ell_{i}+d}, \frac{\lambda_{i}}{\left(c \ell_{i}+d\right)^{2}}\right)_{i=1, \ldots, 3 g} .
$$

3.1.2. Let $\mathcal{E}$ be any bundle of $\operatorname{Bun}_{2}(C)_{\mathcal{O}\left(\sum_{i=1}^{3 g} P_{i}\right)} . T_{\mathcal{E}}^{*} \operatorname{Bun}_{2}(C)_{\mathcal{O}\left(\sum_{i=1}^{3 g} P_{i}\right)}$ is equal to $H^{0}\left(C, \operatorname{End}(\mathcal{E})_{0} \otimes \Omega_{C}\right)$, where $\Omega_{C}$ is the sheaf of differentials on $C$ and $\operatorname{End}(\mathcal{E})_{0}$ is the sheaf of traceless endomorphisms of $\mathcal{E}$.

Lemma 3.1. Let $\mathcal{E}=\mathcal{H}_{\underline{P}}(\underline{\ell})$. Let us define $\Omega(C)$ as the space of rational differentials on $C$. Then $H^{\overline{0}}\left(C, \operatorname{End}(\mathcal{E})_{0} \otimes \Omega_{C}\right)$ is isomorphic with the subspace of $\Omega(C) \otimes \mathfrak{s l}_{2}$ consisting of the elements $A(z)$ such that

i) $A(z)$ is regular on $C \backslash\left\{P_{i}, i=1, \ldots, 3 g\right\}$ and has simple poles at the $P_{i}$,

ii) for any $i=1, \ldots, 3 g$, $\operatorname{res}_{z=P_{i}} A(z)$ belongs to $\mathbb{C}\left(-e+\ell_{i} h+\ell_{i}^{2} f\right)$,

iii) if we set $A(z)=A_{e} \otimes e+A_{h} \otimes h+A_{f} \otimes f$, with $A_{e}, A_{h}, A_{f}$ in $\Omega(C)$, then $-\ell_{i}^{2} A_{e}-2 \ell_{i} A_{h}+A_{f}$ is regular at $P_{i}$, for any $i=1, \ldots, 3 g$.

Condition iii) means that if $\rho$ is the fundamental representation of $\mathfrak{s l}_{2}$, the form $\operatorname{det}\left(\left(\begin{array}{c}1 \\ \ell_{i}\end{array}\right),(\mathrm{id} \otimes \rho)(A(z))\left(\begin{array}{l}1 \\ \ell_{i}\end{array}\right)\right)$ is regular at $P_{i}$.

3.1.3. Let us fix a basis $\left(A_{a}, B_{a}\right)_{a=1, \ldots, g}$ of $a$ - and $b$-cycles on $C$ and let $\left(\omega_{a}\right)_{a=1, \ldots, g}$ be the associated Abelian differentials on $C$.

Let us set $\omega^{(P)}(z)=r^{(z)}(P)$ (see formula (5)).

Lemma 3.2. In terms of the identifications of 3.1.1 and Lemma 3.1, the map $T_{\underline{\ell}}^{*} \mathcal{H}_{\underline{P}}$ is given by

$$
T_{\underline{\ell}}^{*} \mathcal{H}_{\underline{P}}\left(\lambda_{1}, \ldots, \lambda_{3 g}\right)=A(\underline{\ell}, \underline{\lambda} \mid z)
$$

where $A(\underline{\ell}, \underline{\lambda} \mid z)$ is defined by (2)

\subsection{Expression of the Hitchin Hamiltonians.}


3.2.1. The Hitchin fibration is defined as the map

$$
T^{*} \operatorname{Bun}_{2}(C)_{\mathcal{O}\left(\sum_{i} P_{i}\right)} \rightarrow H^{0}\left(C,\left(\Omega_{C}\right)^{\otimes 2}\right),
$$

$(\mathcal{E}, \xi) \mapsto(\mathrm{id} \otimes \operatorname{tr})\left(\xi^{2}\right)$, where $\xi$ belongs to $H^{0}\left(C, \Omega_{C} \otimes \operatorname{End}(\mathcal{E})_{0}\right)$. The Hitchin Hamiltonians are defined as follows. Let $\omega_{1}^{(2)}, \ldots, \omega_{3 g-3}^{(2)}$ be a basis of the space of quadratic differentials on $C$ and let us set

$$
(\mathrm{id} \otimes \operatorname{tr})\left(\xi^{2}\right)=\sum_{\alpha=1}^{3 g-3} \operatorname{Hitch}_{\alpha}(\mathcal{E}, \xi) \omega_{\alpha}^{(2)} ;
$$

the $(\mathcal{E}, \xi) \mapsto \operatorname{Hitch}_{\alpha}(\mathcal{E}, \xi)$ form a Poisson-commutative family of functions on $T^{*} \operatorname{Bun}_{2}(C)_{\mathcal{O}\left(\sum_{i} P_{i}\right)}$, the Hitchin Hamiltonians (see [14).

3.2.2. The right side of (12) is equal to $A(\underline{\ell}, \underline{\lambda} \mid z)$ defined in (2). Prop. 1.1 follows from

$$
H(\underline{\ell}, \underline{\lambda} \mid z)=\operatorname{tr} \rho[A(\underline{\ell}, \underline{\lambda} \mid z)]^{2},
$$

where $\rho$ is the fundamental representation of $\mathfrak{s l}_{2}$.

Another expression of $H(\underline{\ell}, \underline{\lambda} \mid z)$ may be obtained as follows. Let us define $A^{r e g}$ as the second sum of (12), and $\nu_{k i}(z)$ as $\operatorname{det}\left(a_{k i}\right)$, where $a_{k i}$ is the matrix with coefficients $\left(a_{k i}\right)_{j, 3 a-\alpha}=\ell_{j}^{\alpha} \omega_{a}\left(P_{j}\right)$ if $j \neq k$, and $\left(a_{k i}\right)_{k, 3 a-\alpha}=\ell_{i}^{\alpha} \omega_{a}(z)$ if $a=1, \ldots, g, \alpha=1,2,3$. Then

$$
\begin{aligned}
H(\underline{\ell}, \underline{\lambda} \mid z)=\operatorname{tr} \rho\left(A^{r e g}\right)^{2} & +\frac{1}{\operatorname{Den}(\underline{P}, \underline{\ell})} \sum_{i, j, k ; k \neq i} \lambda_{i} \lambda_{j} \ell_{k i}^{2} \nu_{k j}(z) \omega^{\left(P_{i}\right)}\left(P_{k}\right) \omega^{\left(P_{j}\right)}(z) \\
& -\frac{1}{2} \sum_{i, j ; i \neq j} \lambda_{i} \lambda_{j} \ell_{i j}^{2} \omega^{\left(P_{i}\right)}(z) \omega^{\left(P_{j}\right)}(z) .
\end{aligned}
$$

4. Parametrization of Conformal blocks (the Case $N=3 g$ )

\section{1. "Twisting" group elements.}

4.1.1. Element $g_{\underline{\ell}, \underline{P}}$ at level zero. Let $P_{0}$ be a point of $C$, distinct from the $P_{1}, \ldots, P_{3 g}$. Let $J(C)$ be the Jacobian of $C ; J(C)$ is the direct sum $\oplus_{i \in \mathbb{Z}} J^{i}(C)$ of its graded components. Let us denote by $A: C \rightarrow J^{1}(C)$ the Abel-Jacobi map. We assume that

$$
A\left(P_{1}\right)+\ldots+A\left(P_{3 g}\right)=3 g A\left(P_{0}\right) .
$$

(The results of this section can easily be transposed when $3 g P_{0}$ is replaced by an arbitrary effective divisor of degree $3 g$.)

Let us set, for $z$ in $C$,

$$
b_{\underline{P}}(z)=\frac{\prod_{i=1}^{3 g} \Theta\left(A(z)-A\left(P_{i}\right)+\delta-\Delta\right)}{\Theta\left(A(z)-A\left(P_{0}\right)+\delta-\Delta\right)^{3 g}} .
$$

Assumption (13) implies that $b_{P}$ is a rational rational function on $C$, with divisor $\sum_{i=1}^{3 g} P_{i}-3 g P_{0}$ (so $b_{\underline{P}}$ has zeroes at the $P_{i}$ and a pole at $P_{0}$ ). Let us fix functions 
$d_{i, \underline{P}}$, regular on $C \backslash\left\{P_{0}\right\}$, such that $d_{i, \underline{P}}\left(P_{j}\right)=\delta_{i j}$. We then set, for $\ell_{i}$ in $\mathbb{C} P^{1} \backslash\{0\}$, and $z$ in $C \backslash\left\{P_{0}, P_{i}, i=1, \ldots, 3 g\right\}$,

$$
g_{\underline{\ell}, \underline{P}}(z)=\left(\begin{array}{cc}
b_{\underline{P}}^{-1} & 0 \\
0 & 1
\end{array}\right)\left(\begin{array}{cc}
1 & -\sum_{i} \ell_{i}^{-1} d_{i, \underline{P}} \\
0 & 1
\end{array}\right)(z) .
$$

Recall that for $A$ a ring, $G L_{2}(A)$ is defined as the group of matrices $\left(\begin{array}{ll}a & b \\ c & d\end{array}\right)$, with $a, b, c, d$ in $A$, and $a d-b c$ in $A^{\times}\left(A^{\times}\right.$denotes the set of invertible elements of $A$ ). For each point $P$ of $C$, we fix a local coordinate $z_{P}$ at $P$. Recall that $G L_{2}(\mathbb{C}(C))$ may be viewed as a subgroup of the adeles group $G L_{2}\left(\mathbb{A}_{C}\right)=$ $\prod_{P \in C}^{\prime} G L_{2}\left(\mathbb{C}\left(\left(z_{P}\right)\right)\right)$.

Lemma 4.1. For each family $\underline{\ell}=\left(\ell_{i}\right)_{i=1, \ldots, 3 g}$ of $\left(\mathbb{C} P^{1} \backslash\{0\}\right)^{3 g}$, the function $g_{\underline{\ell}, \underline{P}}$ satisfies the equivalent conditions

1) $z \mapsto g_{\underline{\ell}, \underline{P}}(z)$ is a rational function on $C$ with values in $G$, regular on $C \backslash$ $\left\{P_{0} ; P_{i}, i=1, \ldots, 3 g\right\}$, with simple poles at each $P_{i}, i=1, \ldots, 3 g$, the expansion

$$
g_{\underline{\ell}, \underline{P}}(z)=\frac{1}{z_{P_{i}}}\left(\begin{array}{l}
a_{i} \\
c_{i}
\end{array}\right)\left(\begin{array}{ll}
\ell_{i} & -1
\end{array}\right)+O(1)
$$

at $P_{i}$, and $\operatorname{det}\left(g_{\underline{\ell}, \underline{P}}\right)$ has the expansion $\operatorname{det}\left(g_{\underline{\ell}, \underline{P}}\right)=c_{i} z_{P_{i}}^{-1}+O(1)$ at the same point, where $a_{i}, b_{i}$ and $c_{i}$ are complex numbers, such that $\left(a_{i}, b_{i}\right) \neq(0,0)$ and $c_{i} \neq 0$;

2) $g_{\underline{\ell}, \underline{P}}$ belongs to the intersection of $G L_{2}(\mathbb{C}(C))$ and the subset

$G L_{2}\left(\mathbb{C}\left(\left(z_{P_{0}}\right)\right)\right) \times \prod_{i=1}^{3 g} G L_{2}\left(\mathbb{C}\left[\left[z_{P_{i}}\right]\right]\right) \operatorname{diag}\left(1, z_{P_{i}}^{-1}\right)\left(\begin{array}{cc}1 & 0 \\ -\ell_{i} & 1\end{array}\right) \times \prod_{Q \notin\left\{P_{0}, P_{i}\right\}} G L_{2}\left(\mathbb{C}\left[\left[z_{Q}\right]\right]\right)$ of $G L_{2}\left(\mathbb{A}_{C}\right)$.

The properties of $g_{\underline{\ell}, \underline{P}}(z)$ with respect to variation of $\underline{\ell}$ can be described as follows. Let $\mathcal{K}$ be the local field of $C$ at $P_{0}$, so $\mathcal{K}=\mathbb{C}\left(\left(z_{P_{0}}\right)\right)$. Let us set $R=H^{0}\left(C \backslash\left\{P_{0}\right\}, \mathcal{O}_{C}\right)$, which we view as a subring of $\mathcal{K}$, and let us set $\mathfrak{g}^{\text {out }}=\overline{\mathfrak{g}} \otimes R$. It is a Lie subalgebra of $L \overline{\mathfrak{g}}=\overline{\mathfrak{g}} \otimes \mathcal{K}$. Let us denote by $\widetilde{\mathfrak{g}}_{\ell}^{\text {out }}$ the subspace of $L \overline{\mathfrak{g}}$ formed of the Laurent expansions at $P_{0}$ of the rational maps $\phi: C \rightarrow \overline{\mathfrak{g}}$, regular outside $\left\{P_{0}, P_{1}, \ldots, P_{3 g}\right\}$, with expansion at each $P_{i}, i \neq 0$

$$
\phi(z)=\frac{\lambda_{i}}{z_{P_{i}}}\left(-e+\ell_{i} h+\ell_{i}^{2} f\right)+O(1)
$$

where the $\lambda_{i}$ are complex numbers.

Define for $i=1, \ldots, 3 g, \mu_{i}$ as the linear map from $\widetilde{\mathfrak{g}}_{\left(\ell_{i}\right)}^{\text {out }}$ to $\mathbb{C}$ given by

$$
\mu_{i}(\phi)=\left(-\phi_{f}+2 \ell_{i} \phi_{h}+\ell_{i}^{2} \phi_{e}\right)\left(P_{i}\right),
$$

for $\phi=\phi_{e} e+\phi_{h} h+\phi_{f} f, \phi_{e}, \phi_{h}, \phi_{f}$ in $\mathbb{C}(C)$. Let us set $\mathfrak{g}_{\underline{\ell}}^{\text {out }}:=\operatorname{Ad}\left(g_{\underline{\ell}, \underline{P}}\right)^{-1}\left(\mathfrak{g}^{\text {out }}\right)$ (Ad denotes the adjoint action of a group on its Lie algebra). Then we have

$$
\mathfrak{g}_{\underline{\ell}}^{\text {out }}=\operatorname{Ker}\left(\oplus_{i=1}^{3 g} \mu_{i}: \widetilde{\mathfrak{g}}_{\underline{\ell}}^{\text {out }} \rightarrow \mathbb{C}^{3 g}\right) .
$$


Moreover, we have

Lemma 4.2. For any $\phi$ in $\widetilde{\mathfrak{g}}_{\underline{\ell}}^{\text {out }}$, we have

$$
\operatorname{Ad}\left(g_{\underline{\ell}, \underline{P}}\right)(\phi) \in \sum_{i=1}^{3 g} \mu_{i}(\phi)\left(\partial_{\ell_{i}} g_{\underline{\ell}, \underline{P}}\right) g_{\underline{\ell}, \underline{P}}^{-1}+\mathfrak{g}^{\text {out }}
$$

Proof. One first shows this statement for $\phi$ of the form $e \otimes r, r \in R$, using the explicit formula for $g_{\underline{\ell}, \underline{P}}$. In the case of a general $\phi$, one then replaces $\phi$ by $\phi+e \otimes\left\{\sum_{i} \ell_{i}^{-2} \mu_{i}(\phi) d_{i, \underline{P}}\right\}$ and uses (14).

4.1.2. Kac-Moody algebras, extended loop groups and semidirect products. Let $\mathfrak{g}$ be the Kac-Moody Lie algebra $\mathfrak{g}=L \mathfrak{g} \oplus \mathbb{C} K$. It is endowed with the Lie bracket $\left[(x \otimes a, b K),\left(x^{\prime} \otimes a^{\prime}, b^{\prime} K\right)\right]=\left(\left[x, x^{\prime}\right] \otimes a a^{\prime},\left\langle x, x^{\prime}\right\rangle_{\overline{\mathfrak{g}}} \operatorname{res}_{P_{0}}\left(d a \cdot a^{\prime}\right) K\right)$. For $x$ in $\overline{\mathfrak{g}}$ and $\phi$ in $\mathcal{K}$, we will denote $(x \otimes \phi, 0)$ by $x[\phi]$.

Recall that for any $\operatorname{ring} A, G(A)=S L_{2}(A)$ is the group of matrices $\left(\begin{array}{ll}a & b \\ c & d\end{array}\right)$, where $a, b, c, d$ belongs to $A$ and such that $a d-b c=1$.

Let $k$ be an integer $\geq 0$, and let us denote by $\widehat{G(\mathcal{K})}$ the level $k$ central extension of $G(\mathcal{K})$ by $\mathbb{C}^{\times}$(see $[10]$ ). We denote by $Z$ the center of $\widehat{G(\mathcal{K})} . Z$ is isomorphic with $\mathbb{C}^{\times}$; for $c$ in $\mathbb{C}^{\times}$, we denote by $z(c)$ the corresponding element of $Z$.

Let $k$ be an integer $\geq 0$, and let us denote by $\widehat{G(\mathcal{K})}$ the level $k$ central extension of $G^{\prime}(\mathcal{K})$ by $\mathbb{C}^{\times}$(see e.g. $10,[16]$ ). We denote by $Z$ the center of $\widehat{G(\mathcal{K})} . Z$ is isomorphic with $\mathbb{C}^{\times}$; for $c$ in $\mathbb{C}^{\times}$, we denote by $z(c)$ the corresponding element of $Z$.

We will use the following properties of the central extension $\widehat{G(\mathcal{K})}$ :

a) if $\left(\rho_{\mathbb{V}}, \mathbb{V}\right)$ is any integrable $\mathfrak{g}$-module of level $k$, there is a unique lift (denoted $\rho_{\mathbb{V}}^{\text {group }}$ ) of the action of $\mathfrak{g}$ to a representation of $\widehat{G(\mathcal{K})}$ on $\mathbb{V}$; this lift satisfies $\rho_{\mathbb{V}}^{\text {group }}(z(c))=c \mathrm{id}_{\mathbb{V}}$, for $c$ in $\mathbb{C}^{\times}$, and the compatibility rule $\rho_{\mathbb{V}}(\operatorname{Ad}(\pi(g))(x))=$ $\rho_{\mathbb{V}}^{\text {group }}(g) \rho_{\mathbb{V}}(x) \rho_{\mathbb{V}}^{\text {group }}(g)^{-1}$, for any $g$ in $\widehat{G(\mathcal{K})}$ and $x$ in $\mathfrak{g}$, where $\pi$ is the canonical map from $\widehat{G(\mathcal{K})}$ to $G(\mathcal{K})$ and denotes the adjoint action of $G(\mathcal{K})$ on $\mathfrak{g}$;

b) let $N$ be the positive unipotent subgroup of $G$, then there is a unique lift $\iota$ of $N(\mathcal{K})$ to $\widehat{G(\mathcal{K})}$, such that for each formal series $\rho$ of $\mathcal{K}$, we have

$$
\rho_{\mathbb{V}}^{\text {group }}\left(\iota\left(\left(\begin{array}{ll}
1 & \rho \\
0 & 1
\end{array}\right)\right)\right)=\rho_{\mathbb{V}}(\exp (e[\rho])) .
$$

The adjoint actions of $\widehat{G(\mathcal{K})}$ on $\mathfrak{g}$ and on itself factor through actions of $G(\mathcal{K})$ on $\mathfrak{g}$ and on $\widehat{G(\mathcal{K})}$. One can show that these actions extend to actions of $G L_{2}(\mathcal{K})$ 
on these sets, which we denote by Ad. Let us denote by $\widetilde{T}_{\mathfrak{g}}$ and $\widetilde{T}_{\widehat{G(\mathcal{K})}}$ the automorphisms of $\mathfrak{g}$ and of $\widehat{G(\mathcal{K})}$ equal to $\operatorname{Ad}\left(\left(\begin{array}{cc}z_{P_{0}} & 0 \\ 0 & 1\end{array}\right)\right)$. Then $\widetilde{T}_{\mathfrak{g}}$ is given by

$$
\begin{gathered}
\widetilde{T}_{\mathfrak{g}}[(e \otimes \phi, a K)]=\left(e \otimes z_{P_{0}} \phi, a K\right), \widetilde{T}_{\mathfrak{g}}[(f \otimes \phi, a K)]=\left(f \otimes z_{P_{0}}^{-1} \phi, a K\right), \\
\widetilde{T}_{\mathfrak{g}}[(h \otimes \phi, a K)]=\left(h \otimes \phi,\left\{a+\operatorname{res}_{P_{0}}\left(\frac{d z}{z} \phi\right)\right\} K\right),
\end{gathered}
$$

and $\widetilde{T}_{\widehat{G(\mathcal{K})}}$ is the unique group automorphism of $\widehat{G(\mathcal{K})}$ extending the automorphism $\operatorname{Ad}\left(\left(\begin{array}{cc}z_{P_{0}} & 0 \\ 0 & 1\end{array}\right)\right)$ of $G(\mathcal{K})$. Moreover, $\widetilde{T}_{\mathfrak{g}}^{2}$ and $\widetilde{T}_{\widetilde{G(\mathcal{K})}}^{2}$ are both inner automorphisms, equal to $\operatorname{Ad}\left(\begin{array}{cc}z_{P_{0}} & 0 \\ 0 & z_{P_{0}}^{-1}\end{array}\right)$.

Define now $\widehat{G(\mathcal{K})} \rtimes \mathbb{Z}$ as the semidirect product of $\widehat{G(\mathcal{K})}$ with the action of $\mathbb{Z}$ provided by $\widetilde{T}_{\widehat{G(\mathcal{K})}}$. Precisely, $\widehat{G(\mathcal{K})} \rtimes \mathbb{Z}$ is the set $\widehat{G(\mathcal{K})} \times \mathbb{Z}$, endowed with the product

$$
(g, n)\left(g^{\prime}, n^{\prime}\right)=\left(g \widetilde{T}_{\widetilde{G(\mathcal{K})}}^{n}\left(g^{\prime}\right), n+n^{\prime}\right) .
$$

We will write $T_{\widehat{G(\mathcal{K})}}$ for the element $\left(e_{\overparen{G(\mathcal{K})}}, 1\right)$, where $e_{\overparen{G(\mathcal{K})}}$ is the neutral element of $\widehat{G(\mathcal{K})}$, so $(g, n)$ will be equal to $g T_{\widehat{G(\mathcal{K})}}^{n}$. The adjoint action of $\widehat{G(\mathcal{K})}$ on $\mathfrak{g}$ extends to an action of $\widehat{G(\mathcal{K})} \rtimes \mathbb{Z}$ on $\mathfrak{g}$ by Lie algebra automorphisms, which we also denote by $\mathrm{Ad}$; it is defined by $\operatorname{Ad}\left(g T_{\widetilde{G(\mathcal{K})}}^{n}\right)(x)=\operatorname{Ad}(g)\left[\widetilde{T}_{\mathfrak{g}}^{n}(x)\right]$.

Let us now construct representations of the group $\widehat{G(\mathcal{K})} \rtimes \mathbb{Z}$. Let $\left(\rho_{\mathbb{V}}, \mathbb{V}\right)$ be an irreducible integrable representation of $\mathfrak{g}$ of level $k$. Two possibilities occur:

a) if $\rho_{\mathbb{V}} \circ T_{\mathfrak{g}}$ is equivalent to $\rho_{\mathbb{V}}$, let $T_{\mathbb{V}}$ be a linear automorphism of $\mathbb{W}$ such that $\rho_{\mathbb{V}} \circ T=\operatorname{Ad}\left(T_{\mathbb{V}}\right) \circ \rho_{\mathbb{V}}$. We set $\mathbb{W}=\mathbb{V}$ and let $\rho_{\mathbb{W}}$ be the map from $\widehat{G(\mathcal{K})} \rtimes \mathbb{Z}$ to $\operatorname{Aut}_{\mathbb{C}}(\mathbb{W})$, defined by $\rho_{\mathbb{W}}^{\text {group }}\left(g T_{G(\mathcal{K})}^{n}\right)=\rho_{\mathbb{V}}^{\text {group }}(g) \circ T_{\mathbb{V}}^{n}$;

b) if $\rho_{\mathbb{V}} \circ T_{\mathfrak{g}}$ is not equivalent to $\rho_{\mathbb{V}}$, we set $\mathbb{W}=\mathbb{V} \oplus \mathbb{V}$, and we define $\rho_{\mathbb{W}}$ as the map from $\widehat{G(\mathcal{K})} \rtimes \mathbb{Z}$ to $\operatorname{Aut}_{\mathbb{C}}(\mathbb{W})$, defined by

$\rho_{\mathbb{W}}^{\text {group }}\left(g T_{\widetilde{G(\mathcal{K})}}^{n}\right):=\left(\begin{array}{cc}\rho_{\mathbb{V}}^{\text {group }}(g) & 0 \\ 0 & \left(\rho_{\mathbb{V}}^{\text {group }} \circ \widetilde{T}_{\widetilde{G(\mathcal{K})}}^{-1}\right)(g)\end{array}\right)\left(\begin{array}{cc}0 & \rho_{\mathbb{V}}^{\text {group }}\left(\operatorname{diag}\left(z_{P_{0}}, z_{P_{0}}^{-1}\right)\right) \\ \operatorname{id}_{\mathbb{V}} & 0\end{array}\right)^{n}$.

In both cases, $\left(\mathbb{W}, \rho_{\mathbb{W}}^{\text {group }}\right)$ is an irreducible representation of $\widehat{G(\mathcal{K})} \rtimes \mathbb{Z}$.

4.1.3. Definition of $\widetilde{g}_{\underline{\ell}, \underline{P}}$. Identify the function $z \mapsto b_{\underline{P}}(z)$ (sect. 4.1.1) with its image in $\mathcal{K}^{\times}$. We have $b_{\underline{P}}=z_{P_{0}}^{-3 g} b_{0}$, where $b_{0}$ belongs to $\mathbb{C}\left[\left[z_{P_{0}}\right]\right]^{\times}$. $b_{0}$ has two opposite square roots, which belong to $\mathbb{C}\left[\left[z_{P_{0}}\right]\right]^{\times}$. Let us fix one of them, which 
we denote by $b_{0}^{1 / 2}$. Let us fix a lift $\tilde{t}$ in $\widehat{G(\mathcal{K})}$ of $\left(\begin{array}{cc}\left(b_{0}^{1 / 2}\right)^{-1} & 0 \\ 0 & b_{0}^{1 / 2}\end{array}\right)$, and let us set $\widetilde{b}_{\underline{P}}=T_{\widetilde{G(\mathcal{K})}}^{3 g} \widetilde{t}$. Let us finally set, for $\underline{\ell}=\left(\ell_{1}, \ldots, \ell_{3 g}\right)$ in $\left(\mathbb{C}^{\times}\right)^{3 g}$,

$$
\widetilde{g}_{\underline{\ell}, \underline{P}}(z):=\widetilde{b}_{\underline{P}} \cdot \iota\left(\left(\begin{array}{cc}
1 & -\sum_{i} \ell_{i}^{-1} d_{i, \underline{P}} \\
0 & 1
\end{array}\right)\right) \cdot z\left(\left(\prod_{i=1}^{3 g} \ell_{i}\right)^{k}\right),
$$

where the rational function $d_{i, \underline{P}}$ is identified with its image in $\mathcal{K}$.

In the previous section, we studied subspaces $\mathfrak{g}^{\text {out }}, \mathfrak{g}_{\underline{\ell}}^{\text {out }}$ and $\widetilde{\mathfrak{g}}_{\underline{\ell}}^{\text {out }}$ of $\overline{\mathfrak{g}} \otimes \mathcal{K}$. We will embed them in $\mathfrak{g}$ using the following maps:

a) $\mathfrak{g}^{\text {out }}$ is mapped to $\mathfrak{g}$ by the map $x \mapsto(x, 0)$;

b) $\widetilde{\mathfrak{g}}_{\underline{\ell}}^{\text {out }}$ is mapped to $\mathfrak{g}$ by the map

$$
i: \phi=\phi_{e} e+\phi_{h} h+\phi_{f} f \mapsto\left(\phi, \sum_{i}\left(\phi_{h}+\ell_{i} \phi_{e}\right)\left(P_{i}\right) K\right)
$$

c) $\mathfrak{g}_{\underline{\ell}}^{\text {out }}$ is mapped to $\mathfrak{g}$ by the restriction of the previous map.

We will denote by $\mathfrak{g}^{\text {out,ext }}, \widetilde{\mathfrak{g}}_{\underline{\ell}}^{\text {out,ext }}$ and $\mathfrak{g}_{\underline{\ell}}^{\text {out,ext }}$ the images of these maps.

Lemma 4.3. $\mathfrak{g}^{\text {out,ext }}$ and $\mathfrak{g}_{\underline{\ell}}^{\text {out,ext }}$ are Lie subalgebras of $\mathfrak{g}$, isomorphic to $\mathfrak{g}^{\text {out }}$ and $\mathfrak{g}_{\underline{\ell}}^{\text {out }}$ respectively. Moreover, we have

$$
\mathfrak{g}_{\underline{\ell}}^{\text {out }, \text { ext }}=\operatorname{Ad}\left(\widetilde{g}_{\underline{\ell}, \underline{P}}\right)^{-1}\left(\mathfrak{g}^{\text {out }, \text { ext }}\right) .
$$

The analogue of Lemma 4.2 is

Lemma 4.4. For any $\phi$ in $\widetilde{\mathfrak{g}}_{\underline{\ell}}^{\text {out }}$, we have

$$
\operatorname{Ad}\left(\widetilde{g}_{\underline{\ell}, \underline{P}}\right)(i(\phi)) \in \sum_{i=1}^{3 g} \mu_{i}(\phi)\left(\partial_{\ell_{i}} \widetilde{g}_{\underline{\ell}, \underline{P}}\right) \widetilde{g}_{\underline{\ell}, \underline{P}}^{-1}+\mathfrak{g}^{\text {out }, e x t} .
$$

Proof. One again checks this when $\phi=e \otimes \phi$ for $\phi$ in $R$, and uses then Lemma 4.3 .

4.1.4. Properties of $\widetilde{g}_{\underline{\underline{\ell}}, \underline{P}}$. Let us set $G^{\text {out }}=G(R)$. There is unique lift of $G^{\text {out }}$ to a subgroup of $\widehat{G(\mathcal{K})}$, which we denote by $G^{\text {out,ext }}$, corresponding to the Lie algebra inclusion $\mathfrak{g}^{\text {out }, \text { ext }} \subset \mathfrak{g}$. This lift is such that for any integrable representation $\mathbb{V}$, and any $\mathfrak{g}^{\text {out,ext }}$-invariant linear form $\psi$ on $\mathbb{V}, \psi$ is also $G^{\text {out,ext }}$-invariant.

Lemma 4.5. The rational functions $h:\left(\mathbb{C} P^{1}\right)^{3 g} \rightarrow \widehat{G(\mathcal{K})}, \underline{\ell}=\left(\ell_{i}\right)_{i=1, \ldots, 3 g} \mapsto h_{\underline{\ell}}$ satisfying the conclusion of Lemma 4.4 for generic $\underline{\ell}$ are exactly the functions $h_{\underline{\ell}}=z_{0} \cdot \gamma_{\underline{\ell}} \cdot \widetilde{g}_{\underline{\ell}, \underline{P}}$, where $z_{0}$ is a (constant in $\underline{\ell}$ ) element of the center $Z$ of $\widehat{G(\mathcal{K})}$, and $\gamma_{\underline{\ell}}$ is a rational function from $\left(\mathbb{C} P^{1}\right)^{3 g}$ to $G^{\text {out }, e x t}$. 
Corollary 4.1. Let us set, for $g=\left(\begin{array}{ll}a & b \\ c & d\end{array}\right) \in G(\mathbb{C})$ and $\ell$ in $\mathbb{C} P^{1}, g \cdot \ell=\frac{a \ell+b}{c \ell+d}$; $(g, \ell) \mapsto g \cdot \ell$ is the homographic action of $G(\mathbb{C})$ on $\mathbb{C} P^{1}$. Let us denote by $(g, \underline{\ell}) \mapsto g \cdot \underline{\ell}$ the diagonal action of $G(\mathbb{C})$ on $\left(\mathbb{C} P^{1}\right)^{N}$. For $g$ in $G(\mathbb{C})$, let us denote by $\widetilde{g}$ the image of $g$ by the lift map from $G^{\text {out }}$ to $G^{\text {out,ext }}$. Then for any $g$ in $G(\mathbb{C})$, there exists a rational function $\underline{\ell} \mapsto \gamma(g, \underline{\ell})$ with values in $G^{\text {out,ext }}$, such that $\widetilde{g}_{g \cdot \underline{\ell}, \underline{P}}=\gamma(g, \underline{\ell}) \cdot \widetilde{g}_{\underline{\ell}, \underline{P}} \cdot \widetilde{g} \cdot z\left(\prod_{i=1}^{3 g}\left(c \ell_{i}+d\right)^{-k}\right)$.

Proof. Let us set $h_{g, \underline{\ell}}:=\widetilde{g}_{g \cdot \underline{\ell}, \underline{P}} \cdot \widetilde{g}^{-1} \cdot z\left(\prod_{i=1}^{3 g}\left(c \ell_{i}+d\right)^{k}\right)$. Then one checks that $h_{g, \underline{\ell}}$ satisfies the conclusion of Lemma 4.4. Then Lemma 4.5 implies that there exists an element $z_{g}$ of $Z$ and a rational function $\underline{\ell} \mapsto \gamma(g, \underline{\ell})$ with values in $G^{\text {out,ext }}$, such that $h_{g, \underline{\ell}}=z_{g} \cdot \gamma(g, \underline{\ell}) \cdot \widetilde{g}_{\underline{\ell}, \underline{P}} \cdot g \mapsto z_{g}$ is therefore a group homomorphism from $G(\mathbb{C})$ to $Z=\mathbb{C}^{\times}$, and is therefore trivial.

Remark 3. It is easy to check directly Lemma 4.1 when $g$ is lower-triangular.

4.2. Conformal blocks and associated functions. Let $k$ be an integer $\geq 0$ and let $\mathbb{W}$ be one of the level $k$ representations of $\widehat{G(\mathcal{K})} \rtimes \mathbb{Z}$ constructed in sect. 4.1.2. For any integer $a \geq 0$, let $\left(\rho_{a}, V_{a}\right)$ be the irreducible $(a+1)$-dimensional representation of $\overline{\mathfrak{g}}$. The irreducible integrable representations of level $k$ are the $\mathbb{V}_{a, k}$, where $0 \leq a \leq k . \mathbb{V}_{a, k}$ may be constructed as the quotient of the Weyl module $U \mathfrak{g} \otimes_{U \mathfrak{g}_{+}} V_{a}$, where $\mathfrak{g}_{+}=\overline{\mathfrak{g}} \otimes \mathbb{C}[[t]] \oplus \mathbb{C} K$ and $V_{a}$ is endowed with the $\mathfrak{g}_{+}$-module structure defined by $\rho_{a,+}: \mathfrak{g}_{+} \rightarrow \operatorname{End}\left(V_{a}\right)$ such that $\rho_{a,+}(x \otimes f, a K)=$ $\rho_{a}(x) f(0)+k a \mathrm{id}_{V_{a}}$.

So

a) either $k$ is even and $\mathbb{W}=\mathbb{V}_{k / 2, k}$;

b) or we have, for $a \neq b$ and $a+b=k, \mathbb{W}=\mathbb{V}_{a, k} \oplus \mathbb{V}_{b, k}$.

We denote by $W$ the subspace of $\mathbb{W}$ equal to the images of the subspaces of the Weyl modules $1 \otimes V_{k / 2}$ in case a), and $\left(1 \otimes V_{a}\right) \oplus\left(1 \otimes V_{b}\right)$ in case b); so $W$ is isomorphic to $V_{k / 2}$ in case a), and to $V_{a} \oplus V_{b}$ in case b). In each case, $W$ is equal to the subspace of $\mathbb{W}$ formed of the elements annihilated by $\mathfrak{g}^{i n}$.

Let $\psi$ be a $\mathfrak{g}^{\text {out }}$-invariant linear form on $\mathbb{W}$. We associate to it the function

$$
f_{\psi}(\underline{\ell}, \underline{P} \mid v):=\left\langle\psi, \rho_{\mathbb{W}}^{\text {group }}\left(\widetilde{g}_{\underline{\ell}, \underline{P}}\right) v\right\rangle
$$

where $v$ is in $W$. For any $\underline{P}, f_{\psi}(\underline{\ell}, \underline{P} \mid v)$ belongs therefore to $\otimes_{i=1}^{k}\left\{\ell_{i}^{k} \mathbb{C}\left[\left[\ell_{i}^{-1}\right]\right]\right\} \otimes W^{*}$.

Let us denote by $\mathbb{C}[\ell]_{\leq k}$ the space of polynomials in $\ell$ of degree $\leq k$. This space is endowed with the right $G(\mathbb{C})$-module structure defined by $(g \cdot f)(\ell):=$ $f(g \cdot \ell)(c z+d)^{k}$.

Proof of Prop. 1.9. It follows from its definition that the map $v \mapsto f_{\psi}(\underline{\ell} \mid v)$ belongs to $\operatorname{Hom}_{\mathbb{C}}\left(W, \otimes_{i=1}^{3 g} \ell_{i}^{k} \mathbb{C}\left[\left[\ell_{i}^{-1}\right]\right]\right)$. Let may assume that $v$ is a weight vector, so $h[1] v=\lambda v$, where $\lambda$ is an integer in $\{-k, \ldots, k\}$. Moreover,

$$
f_{\psi}(\underline{\ell}, \underline{P} \mid v)=\prod_{i=1}^{g} \ell_{i}^{k} \sum_{\alpha \geq 0} \frac{1}{\alpha !}\left\langle\psi, \rho_{\mathbb{W}}^{\text {group }}\left(\widetilde{b}_{\underline{P}}\right) \rho_{\mathbb{W}}\left(e\left[\sum_{i} d_{i, \underline{P}} \ell_{i}^{-1}\right]\right)^{\alpha} v\right\rangle .
$$


Since $\rho_{\mathbb{W}}(h[1]) \rho_{\mathbb{V}}^{\text {group }}\left(\widetilde{b}_{\underline{P}}\right)=\rho_{\mathbb{W}}^{\text {group }}\left(\widetilde{b}_{\underline{P}}\right)\left[\rho_{\mathbb{W}}(h[1])-3 g k\right]$, all contributions to this series where $\alpha \neq \frac{3 g k+\lambda}{2}$ are zero. It follows that

$$
f_{\psi}(\underline{\ell}, \underline{P} \mid v)=\frac{1}{\alpha !}\left\langle\psi, \rho_{\mathbb{W}}^{\text {group }}\left(\widetilde{b}_{\underline{P}}\right) \rho_{\mathbb{W}}\left(e\left[\sum_{i} d_{i, \underline{P}} \ell_{i}^{-1}\right]\right)^{\alpha} v\right\rangle, \quad \text { with } \quad \alpha=\frac{3 g k+\lambda}{2} .
$$

Therefore, for any $v$ in $W, f_{\psi}(\underline{\ell}, \underline{P} \mid v)$ is a Laurent polynomial in the $\ell_{i}$, of degree in each $\ell_{i}$ smaller than $k$.

Finally, Cor. 4.1, together with the fact that $\rho_{\mathbb{W}}^{\text {group }}(\widetilde{g}) v=\rho_{W}(g) v$ for $g \in G(\mathbb{C})$, imply that $f_{\psi}(g \cdot \underline{\ell}, \underline{P} \mid v)=\prod_{i=1}^{3 g}\left(c \ell_{i}+d\right)^{k} f_{\psi}\left(\underline{\ell}, \underline{P} \mid \rho_{W}(g) v\right)$, for $g=\left(\begin{array}{ll}a & b \\ c & d\end{array}\right) \in G(\mathbb{C})$. This formula both shows that $v \mapsto f_{\psi}(\underline{\ell}, \underline{P} \mid v)$ is polynomial in the $\ell_{i}$, and that it is a morphism of $\overline{\mathfrak{g}}$-modules.

We also get

Proposition 4.1. If $\mathbb{W}$ is a representation described of type b) above, and if $\psi$ is zero when restricted to $\mathbb{V}_{b, k}, f_{\psi}(\underline{\ell}, \underline{P} \mid v)$ is zero when $v$ belongs to $V_{a}$ if the genus $g$ of $C$ is odd, and when $v$ belongs to $V_{b}$ if $g$ is even.

Remark 4 . The fact that $\mathbb{W}$ is integrable should imply some additional functional properties of $f_{\psi}(\underline{\ell}, \underline{P} \mid v)$.

Remark 5. It follows from Lemma 4.5 that if we replace, in the definition of $f_{\psi}(\underline{\ell}, \underline{P} \mid v), \widetilde{g}_{\underline{\ell}, \underline{P}}$ by any function $h_{\underline{\ell}, \underline{P}}$ satisfing the conclusions of Lemma 4.4 , the function $f_{\psi}(\underline{\underline{\ell}}, \underline{P} \mid v)$ only gets multiplied by a nonzero function of $\underline{P}$. Moreover, since for any irreducible integrable representation $\mathbb{V}$, and any lift of $\left(\begin{array}{cc}-1 & 0 \\ 0 & -1\end{array}\right) \in$ $G(\mathcal{K})$, its image by $\rho_{\mathbb{V}}^{\text {group }}$ is scalar, a change in the choice of the square root $b_{0}^{1 / 2}$ of $b_{0}$ only has the effect of multiplying $f_{\psi}(\underline{\ell}, \underline{P} \mid v)$ by a nonzero function of $\underline{P}$.

\section{Action of the Sugawara tensor}

5.1. Expressions of the Sugawara tensor $T(z)$. For $x$ in $\overline{\mathfrak{g}}$ and $\phi$ in $\mathcal{K}$, let us denote by $x[\phi]$ the element $(x \otimes \phi, 0)$ of $\mathfrak{g}$.

Let Cas be the element of $\overline{\mathfrak{g}} \otimes \overline{\mathfrak{g}}$ corresponding to $\langle,\rangle_{\overline{\mathfrak{g}}}$. We have Cas $=\frac{1}{2} h \otimes$ $h+e \otimes f+f \otimes e$.

Let us set for $x=e, h, f, x(z)=\sum_{i \in \mathbb{Z}} x\left[z_{P_{0}}^{i}\right] z^{-i-1} d z$. We define the Green function $G(z, w) d z$ as

$$
G(z, w) d z=d_{z} \ln \Theta(A(z)-A(w)+\delta-\Delta)-d_{z} \ln \Theta\left(A(z)-A\left(P_{0}\right)+\delta-\Delta\right) .
$$

We have then the expansion at the diagonal

$$
G(z, w) d z=\frac{d z}{z-w}+O(1) .
$$


HECKE-TYURIN PARAMETRIZATION OF THE HITCHIN AND KZB SYSTEMS

Expand $G(z, w) d z$ as $\sum_{i>0} \alpha_{i}(z) w^{i}$ for $w \ll z$ and as $\sum_{i>0} z^{i-1} d z \beta_{i}(w)$ for $z \ll w$ (both $z$ and $w$ being at the formal neighborhood of $P_{0}$ ). Let us then set

$$
x^{i n}(z)=\sum_{i>0} x\left[w^{i}\right] \alpha_{i}(z), \quad x^{o u t}(z)=\sum_{i>0} x\left[\beta_{i}\right] z^{i-1} d z
$$

$\left(x^{\text {in }}(z)\right.$ and $x^{\text {out }}(z)$ therefore belong to $\left.\mathfrak{g} \hat{\otimes} \Omega_{z}\right)$ and set

$$
T(z)=\frac{1}{2 \kappa} \sum_{\alpha} x_{\alpha}^{o u t}(z) y_{\alpha}(z)+y_{\alpha}(z) x_{\alpha}^{\text {in }}(z),
$$

where $\kappa=k+2$, and $\sum_{\alpha} x_{\alpha} \otimes y_{\alpha}=$ Cas. The Laurent coefficients of $T(z)$ belong to a completion of the quotient $U \mathfrak{g} /(K-k \cdot 1)$ of the enveloping algebra of $\mathfrak{g}$.

The following Lemma shows that $T(z)$ is a normalization of the Sugawara tensor.

Lemma 5.1. In any highest weight $\mathfrak{g}$-module, the matrix elements of $T(z)$ coincide with those of

$$
\frac{1}{2 \kappa} \lim _{z^{\prime} \rightarrow z}\left(\sum_{\alpha} x_{\alpha}(z) y_{\alpha}\left(z^{\prime}\right)-3 d_{z^{\prime}}\left[G\left(z, z^{\prime}\right) d z\right]\right) .
$$

We may also write (15) as follows. Let us set

$$
\ell^{\text {in }, \text { std }}(z)=\sum_{\alpha} x_{\alpha} \otimes y_{\alpha}^{\text {in }}(z), \ell^{\text {out }, \text { std }}(z)=\sum_{\alpha} x_{\alpha} \otimes y_{\alpha}^{\text {out }}(z), \ell(z)=\ell^{\text {in }, \text { std }}(z)+\ell^{\text {out }, \text { std }}(z) .
$$

Then

$$
T(z)=\frac{1}{2 \kappa}[(\operatorname{tr} \circ \rho) \otimes \mathrm{id}]\left\{\ell^{o u t, s t d}(z) \ell(z)+\ell(z) \ell^{i n, s t d}(z)\right\} .
$$

Let $\lambda(z)=\sum_{\alpha} x_{\alpha} \otimes \lambda_{\alpha}(z)$ be an element of $(L \overline{\mathfrak{g}} \otimes \Omega) \otimes \overline{\mathfrak{g}}$ and let us set now

$$
\ell_{\underline{\ell}}^{\text {in }}(z)=\ell^{\text {in,std }}(z)+\lambda(z), \quad \ell_{\underline{\ell}}^{\text {out }}(z)=\ell^{\text {out }, \text { std }}(z)-\lambda(z) .
$$

Then

$$
\begin{aligned}
T(z) & \left.=\frac{1}{2 \kappa}[(\operatorname{tr} \circ \rho) \otimes \mathrm{id}]\left\{\ell_{\underline{\ell}}^{\text {out }}(z) \ell(z)+\ell(z) \ell_{\underline{\ell}}^{\text {in }}(z)\right\}-[\lambda(z), \ell(z)]\right\} \\
& =\frac{1}{2 \kappa}\left([\mathrm{id} \otimes(\operatorname{tr} \circ \rho)]\left\{\ell_{\underline{\ell}}^{\text {out }}(z) \ell(z)+\ell(z) \ell_{\underline{\ell}}^{\text {in }}(z)\right\}-\sum_{\alpha}\left[\lambda_{\alpha}(z), x_{\alpha}(z)\right]\right) .
\end{aligned}
$$

5.2. Decomposition of $L \overline{\mathfrak{g}}$. Let us define $\mathfrak{g}^{\text {in }}$ as the Lie subalgebra of $L \overline{\mathfrak{g}}$ equal to $t \overline{\mathfrak{g}}[[t]]$. Then

Lemma 5.2. If $\operatorname{Den}(\underline{P}, \underline{\ell}) \neq 0$, we have a direct sum decomposition

$$
L \overline{\mathfrak{g}}=\widetilde{\mathfrak{g}}_{\underline{\ell}}^{\text {out }} \oplus \mathfrak{g}^{\text {in }} .
$$


Proof. $\widetilde{\mathfrak{g}}_{\underline{\ell}}^{\text {out }}$ is the space of all sums

$$
y(z)+\sum_{i} A_{i}\left(-e+\ell_{i} h+\ell_{i}^{2} f\right)\left[r^{\left(P_{i}\right)}(z)-r^{\left(P_{0}\right)}(z)\right],
$$

where $z \mapsto y(z)$ is a regular map from $C \backslash\left\{P_{0}\right\}$ to $\overline{\mathfrak{g}}$, and the $A_{i}$ are tangent vectors at $P_{i}$.

The intersection $\widetilde{\mathfrak{g}}_{\ell}^{\text {out }} \cap \overline{\mathfrak{g}}[[t]]$ is therefore equal to the space of vectors of the form (18), such that $y(z)-\left[\sum_{i} A_{i}\left(-e+\ell_{i} h+\ell_{i}^{2} f\right)\right] r^{\left(P_{0}\right)}(z)$ is regular at $P_{0}$. Since there is no nonconstant function $v$ on the universal cover $\widetilde{C}$ of $C$, regular everywhere and with transformation properties $v \circ \gamma_{A_{a}}=v, v \circ \gamma_{A_{a}}-v=$ constant, $y$ should be constant and $\sum_{i} A_{i}\left(-e+\ell_{i} h+\ell_{i}^{2} f\right)$ should vanish. Now the equations

$$
\sum_{i} A_{i} \omega_{a}\left(P_{i}\right)=\sum_{i} A_{i} \omega_{a}\left(P_{i}\right) \ell_{i}=\sum_{i} A_{i} \omega_{a}\left(P_{i}\right) \ell_{i}^{2}=0
$$

imply that the $A_{i}$ are all zero. Therefore $\widetilde{\mathfrak{g}}_{\underline{\ell}}^{\text {out }} \cap \overline{\mathfrak{g}}[[t]]=\overline{\mathfrak{g}}$, so $\widetilde{\mathfrak{g}}_{\underline{\ell}}^{\text {out }} \cap \mathfrak{g}^{\text {in }}=0$.

Let us now prove that $\widetilde{\mathfrak{g}}_{\underline{\ell}}^{\text {out }}+\mathfrak{g}^{\text {in }}=L \overline{\mathfrak{g}}$. We have

$$
L \overline{\mathfrak{g}} /\left(\mathfrak{g}^{\text {in }}+\mathfrak{g}_{\underline{\ell}}^{\text {out }}\right)=H^{1}\left(C, \operatorname{End}(\mathcal{E})_{0}(-P)\right),
$$

and since $\mathcal{E}$ is stable, we have $\operatorname{dim} H^{1}\left(C, \operatorname{End}(\mathcal{E})_{0}(-P)\right)=3 g$, so

$$
\operatorname{dim} L \overline{\mathfrak{g}} /\left(\mathfrak{g}^{\text {in }}+\mathfrak{g}_{\underline{\ell}}^{\text {out }}\right)=3 g .
$$

On the other hand, we have $\operatorname{dim} \widetilde{\mathfrak{g}}_{\underline{\ell}}^{\text {out }} / \mathfrak{g}_{\underline{\ell}}^{\text {out }}=3 \mathrm{~g}$. Since the sum of $\mathfrak{g}^{\text {in }}$ and $\widetilde{\mathfrak{g}}_{\underline{\ell}}^{\text {out }}$ is direct, we have also $\operatorname{dim}\left(\mathfrak{g}^{\text {in }}+\widetilde{\mathfrak{g}}_{\underline{\ell}}^{\text {out }}\right) /\left(\mathfrak{g}^{\text {in }}+\mathfrak{g}_{\underline{\ell}}^{\text {out }}\right)=3 g$. Together with (19), this shows $\left.L \overline{\mathfrak{g}}=\mathfrak{g}^{\text {in }}+\widetilde{\mathfrak{g}}_{\underline{\ell}}^{\text {out }}\right)$.

5.3. The $\ell$-operators. Let us set $\mathfrak{g}_{\Omega}=\overline{\mathfrak{g}} \otimes \mathbb{C}((t)) d t$. There is a pairing $\langle,\rangle_{L \overline{\mathfrak{g}} \times \mathfrak{g}_{\Omega}}$ : $L \overline{\mathfrak{g}} \times \mathfrak{g}_{\Omega} \rightarrow \mathbb{C}$, defined as the tensor product of $\langle,\rangle_{\overline{\mathfrak{g}}}$ and the residue pairing at $P_{0}$.

Let us set $\delta(z, w) d z=\sum_{i \in \mathbb{Z}} z^{i} w^{-i-1} d z$. Then the canonical element $L \overline{\mathfrak{g}} \hat{\otimes} \mathfrak{g}_{\Omega}$ is sent by the isomorphism $\mathfrak{g}_{\Omega} \hat{\otimes} L \overline{\mathfrak{g}} \rightarrow \overline{\mathfrak{g}}^{\otimes 2} \otimes \mathbb{C}\left[\left[z^{ \pm 1}, w^{ \pm 1}\right]\right] d z$ to $\ell^{\text {tot }}(z, w) d z=$ $\operatorname{Cas} \delta(z, w) d z$.

We define $\ell_{\underline{\ell}}^{\text {in }}(z, w) d z$ and $\ell_{\underline{\ell}}^{\text {out }}(z, w) d z$ as the images in $\mathfrak{g}_{\Omega} \otimes L \overline{\mathfrak{g}}$ of the canonical elements of $\left(\widetilde{\mathfrak{g}}_{\underline{\ell}}^{\text {out }}\right)^{\perp} \hat{\otimes} \mathfrak{g}^{\text {in }}$ and $\left(\mathfrak{g}^{\text {in }}\right)^{\perp} \hat{\otimes} \widetilde{\mathfrak{g}}_{\underline{\ell}}^{\text {out }}$.

Proposition 5.1. Let us set

$$
\begin{aligned}
& \ell_{\underline{\ell}}^{r a t}(z, w) d z=\operatorname{Cas} G(z, w) d z \\
& -\frac{1}{\operatorname{Den}(\underline{P}, \underline{\ell})} \sum_{i=1}^{3 g}\left[-\partial_{\ell_{i}}^{2} \operatorname{Den}(\underline{P}, \underline{\ell})_{\mid \ell_{i}=0} e+\frac{1}{2} \partial_{\ell_{i}} \operatorname{Den}(\underline{P}, \underline{\ell})_{\mid \ell_{i}=0} h+\frac{1}{2} \operatorname{Den}(\underline{P}, \underline{\ell})_{\mid \ell_{i}=0} f\right]_{\mid P_{i}=z} \\
& \otimes\left(-e+\ell_{i} h+\ell_{i}^{2} f\right) G\left(P_{i}, w\right) d P_{i} \\
& \text { If } f(z, w) d z \text { is a rational form on } C \times C, \text { let us denote by } f(z, w) d z \mid z \ll w \text { and } \\
& f(z, w) d z \mid w \ll z \text { its formal expansions for } z, w \text { in the neighborhood of } P_{0} \text { with } z \ll w,
\end{aligned}
$$


resp. $w \ll z$. Then we have

$$
\ell_{\underline{\ell}}^{o u t}(z, w) d z=-\ell_{\underline{\ell}}^{r a t}(z, w) d z\left|z \ll w, \quad \ell_{\underline{\ell}}^{i n}(z, w) d z=\ell_{\underline{\ell}}^{r a t}(z, w) d z\right| w \ll z .
$$

5.4. Expression of $T(z)$ in terms of $\ell$-operators. We will denote by $\iota$ the embedding of $\left[\overline{\mathfrak{g}} \otimes \Omega_{z}\right] \hat{\otimes}[\overline{\mathfrak{g}} \otimes \mathbb{C}((w))]$ in $\overline{\mathfrak{g}} \otimes\left[\mathfrak{g} \hat{\otimes} \Omega_{z}\right]$ by sending $[a \otimes \omega(z)] \otimes[b \otimes f(w)]$ to $a \otimes[(b \otimes f, 0) \otimes \omega]$. (So $\iota$ preserves the order in the tensor product $\overline{\mathfrak{g}} \otimes \overline{\mathfrak{g}}$, but exchanges variables $z$ and $w$.)

Let us denote by $\ell_{\underline{\ell}}^{\text {in }}(z)$ and $\ell_{\underline{\ell}}^{\text {out }}(z)$ the images of $\ell_{\underline{\ell}}^{\text {in }}(z, w) d z$ and $\ell_{\underline{\ell}}^{\text {out }}(z, w) d z$ by $\iota$. We have $\ell_{\underline{\ell}}^{\text {in }}(z)+\ell_{\underline{\ell}}^{\text {out }}(z)=\ell(z)$.

Let us define $\bar{\lambda}(z)$ and the image by $\iota$ of $\ell_{\underline{\ell}}^{\text {rat }}(z, w) d z$ - Cas $G(z, w) d z$ and define $\lambda_{\alpha}(z)$ by $\lambda(z)=\sum_{\alpha} \lambda_{\alpha}(z) \otimes x_{\alpha}$. Write also $\lambda(z)=\sum_{u, v, \omega, \phi} u[\phi] \omega(z) \otimes v$. Then

$$
\sum_{\alpha}\left[\lambda_{\alpha}(z), x_{\alpha}(z)\right]=\sum \omega(z) \phi(z)[u, v](z)+K \sum \omega(z) d \phi(z)\langle u, v\rangle_{\overline{\mathfrak{g}}} .
$$

Therefore

$$
\begin{aligned}
& \sum_{\alpha}\left[\lambda_{\alpha}(z), x_{\alpha}(z)\right] \\
& =-\frac{1}{\operatorname{Den}(\underline{P}, \underline{\ell})}\left\{e(z) \sum_{i}\left[-\partial_{\ell_{i}} \operatorname{Den}(\underline{P}, \underline{\ell})_{\mid \ell_{i}=0}+2 \ell_{i} \partial_{\ell_{i}}^{2} \operatorname{Den}(\underline{P}, \underline{\ell})_{\mid \ell_{i}=0}\right]_{P_{i}=z} G\left(P_{i}, z\right) d P_{i}\right. \\
& +h(z) \sum_{i}\left[\frac{1}{2} \operatorname{Den}(\underline{P}, \underline{\ell})_{\mid \ell_{i}=0}-\ell_{i}^{2} \partial_{\ell_{i}}^{2} \operatorname{Den}(\underline{P}, \underline{\ell})_{\mid \ell_{i}=0}\right]_{P_{i}=z} G\left(P_{i}, z\right) d P_{i} \\
& \left.+f(z) \sum_{i}\left[\ell_{i} \operatorname{Den}(\underline{P}, \underline{\ell})_{\mid \ell_{i}=0}-\ell_{i}^{2} \partial_{\ell_{i}} \operatorname{Den}(\underline{P}, \underline{\ell})_{\mid \ell_{i}=0}\right]_{P_{i}=z} G\left(P_{i}, z\right) d P_{i}\right\} \\
& -\frac{k}{\operatorname{Den}(\underline{P}, \underline{\ell})} \sum_{i} d_{z}\left[G\left(P_{i}, z\right) d P_{i}\right] \\
& \left\{-\frac{1}{2} \operatorname{Den}(\underline{P}, \underline{\ell})_{\mid \ell_{i}=0}+\ell_{i} \partial_{\ell_{i}} \operatorname{Den}(\underline{P}, \underline{\ell})_{\mid \ell_{i}=0}-\ell_{i}^{2} \partial_{\ell_{i}}^{2} \operatorname{Den}(\underline{P}, \underline{\ell})_{\mid \ell_{i}=0}\right\}_{P_{i}=z} .
\end{aligned}
$$

(17) then yields the following expression of the Sugawara tensor:

Lemma 5.3. We have

$$
T(z)=\frac{1}{2 \kappa}[(\operatorname{tr} \circ \rho) \otimes \mathrm{id}]\left(\ell_{\underline{\underline{e}}}^{\text {out }}(z) \ell(z)+\ell(z) \ell_{\underline{\underline{\ell}}}^{\text {in }}(z)+a_{\underline{P}}(\underline{\ell}, z) \ell(z)\right)+\frac{1}{2 \kappa} s_{\underline{P}}(\underline{\ell}, z),
$$

where $a_{\underline{P}}(\underline{\ell}, z)$ is the element of $\overline{\mathfrak{g}} \otimes \mathbb{C}\left[\ell_{i}, \partial_{\ell_{i}}, i=1, \ldots, 3 g\right]\left[\operatorname{Den}(\underline{P}, \underline{\ell})^{-1}\right] \otimes \Omega(C)$ equal to

$$
\begin{aligned}
& a_{\underline{P}}(\underline{\ell}, z)=-\frac{1}{\operatorname{Den}(\underline{P}, \underline{\ell})} \sum_{i} G\left(P_{i}, z\right) d P_{i}\left\{\left(\frac{1}{2} h+\ell_{i} f\right) \operatorname{Den}(\underline{P}, \underline{\ell})_{\mid \ell_{i}=0}\right. \\
& \left.-\left(e+\ell_{i}^{2} f\right) \partial_{\ell_{i}} \operatorname{Den}(\underline{P}, \underline{\ell})_{\mid \ell_{i}=0}+\left(2 \ell_{i} e-\ell_{i}^{2} h\right) \partial_{\ell_{i}}^{2} \operatorname{Den}(\underline{P}, \underline{\ell})_{\mid \ell_{i}=0}\right\}_{P_{i}=z},
\end{aligned}
$$


and $s_{\underline{P}}(\underline{\ell}, z)$ is the element of $\mathbb{C}\left[\ell_{i}, \partial_{\ell_{i}}, i=1, \ldots, 3 g\right]\left[\operatorname{Den}(\underline{P}, \underline{\ell})^{-1}\right] \otimes \Omega^{2}(C)\left(\Omega^{2}(C)\right.$ is the space of rational sections of $\Omega_{C}^{\otimes 2}$ ) given by

$$
\begin{aligned}
s_{\underline{P}}(\underline{\ell}, z)= & \frac{k}{\operatorname{Den}(\underline{P}, \underline{\ell})} \sum_{i=1}^{3 g} d_{z}\left[G\left(P_{i}, z\right) d P_{i}\right] \\
& \left\{-\frac{1}{2} \operatorname{Den}(\underline{P}, \underline{\ell})_{\mid \ell_{i}=0}+\ell_{i} \partial_{\ell_{i}} \operatorname{Den}(\underline{P}, \underline{\ell})_{\mid \ell_{i}=0}-\ell_{i}^{2} \partial_{\ell_{i}}^{2} \operatorname{Den}(\underline{P}, \underline{\ell})_{\mid \ell_{i}=0}\right\}_{P_{i}=z} .
\end{aligned}
$$

5.5. For $x=x_{e} \otimes e+x_{h} \otimes h+x_{f} \otimes f$ an element of $\widetilde{\mathfrak{g}}_{\left(\ell_{i}\right)}^{\text {out }}$, let us set

$$
\nu(x)=\sum_{i}\left(x_{h}+\ell_{i} x_{e}\right)\left(P_{i}\right) .
$$

We have then

Lemma 5.4. For $v^{\prime}$ any vector $\mathbb{W}$, and $x$ in $\widetilde{\mathfrak{g}}_{\left(\ell_{i}\right)}^{\text {out }}$, we have

$$
\left\langle\psi, \rho_{\mathbb{W}}^{\text {group }}\left(\widetilde{g}_{\underline{\ell}, \underline{P}}\right) \rho_{\mathbb{W}}[(x, 0)] v^{\prime}\right\rangle=\left[\sum_{i=1}^{3 g} \mu_{i}(x) \partial_{\ell_{i}}-k \nu(x)\right]\left\langle\psi, \rho_{\mathbb{W}}^{\text {group }}\left(\widetilde{g}_{\underline{\ell}, \underline{P}}\right) v^{\prime}\right\rangle .
$$

Recall that $\ell_{\underline{\ell}}^{\text {out }}(z, w) d z$ belongs to $\mathfrak{g}_{\Omega} \hat{\otimes} L \overline{\mathfrak{g}}$. The expressions

$$
\mu_{i, \underline{P}}(\underline{\ell}, z):=\left(\mathrm{id} \otimes \mu_{i}\right)\left(\ell_{\underline{\ell}}^{\text {out }}(z, w) d z\right) \text { and } \nu_{\underline{P}}(\underline{\ell}, z):=(\mathrm{id} \otimes \nu)\left(\ell_{\underline{\ell}}^{\text {out }}(z, w) d z\right)
$$

make sense and belong to $\overline{\mathfrak{g}} \otimes \mathbb{C}\left[\ell_{i}, i=1, \ldots, 3 g\right]\left[\operatorname{Den}(\underline{P}, \underline{\ell})^{-1}\right] \otimes \Omega(C)$. We find $\mu_{i, \underline{P}}(\underline{\ell}, z)=\left(e-\ell_{i} h-\ell_{i}^{2} f\right) G\left(z, P_{i}\right) d z+\frac{1}{\operatorname{Den}(\underline{P}, \underline{\ell})}$.

$\cdot \sum_{j \neq i} \ell_{i j}^{2}\left[\partial_{\ell_{j}}^{2} \operatorname{Den}(\underline{P}, \underline{\ell})_{\mid \ell_{j}=0} e-\frac{1}{2} \partial_{\ell_{j}} \operatorname{Den}(\underline{P}, \underline{\ell})_{\mid \ell_{j}=0} h-\frac{1}{2} \operatorname{Den}(\underline{P}, \underline{\ell})_{\mid \ell_{j}=0} f\right]_{P_{j}=z} G\left(P_{j}, P_{i}\right) d P_{j}$

and

$$
\begin{aligned}
& \nu_{\underline{P}}(\underline{\ell}, z)=-\sum_{i}\left(\frac{1}{2} h+\ell_{i} f\right) G\left(z, P_{i}\right) d z+\frac{1}{\operatorname{Den}(\underline{P}, \underline{\ell})} \cdot \\
& \cdot \sum_{i, j ; i \neq j} \ell_{j i}\left[-\partial_{\ell_{j}}^{2} \operatorname{Den}(\underline{P}, \underline{\ell})_{\mid \ell_{j}=0} e+\frac{1}{2} \partial_{\ell_{j}} \operatorname{Den}(\underline{P}, \underline{\ell})_{\mid \ell_{j}=0} h+\frac{1}{2} \operatorname{Den}(\underline{P}, \underline{\ell})_{\mid \ell_{j}=0} f\right]_{P_{j}=z} G\left(P_{j}, P_{i}\right) d P_{j} .
\end{aligned}
$$

Lemma 5.5. Let us set

$$
\ell_{\underline{P}}^{\text {diff }}(z):=\sum_{i} \mu_{i, \underline{P}}(\underline{\ell}, z) \partial_{\ell_{i}}-k \nu_{\underline{P}}(\underline{\ell}, z) .
$$

$\ell_{\underline{P}}^{\text {diff }}(z)$ is an element of $\overline{\mathfrak{g}} \otimes \mathbb{C}\left[\ell_{i}, \partial_{\ell_{i}}, i=1, \ldots, 3 g\right]\left[\operatorname{Den}(\underline{P}, \underline{\ell})^{-1}\right] \otimes \Omega(C)$, and we have, for any vector $v^{\prime}$ of $\mathbb{W}$,

$$
\left\langle\psi, \rho_{\mathbb{V}}^{\text {group }}\left(\widetilde{g}_{\underline{\ell}, \underline{P}}\right)\left(\mathrm{id} \otimes \rho_{\mathbb{V}}\right)\left(\ell_{\underline{\ell}}^{\text {out }}(z)\right) v^{\prime}\right\rangle=\ell_{\underline{P}}^{\text {diff }}(z)\left\{\left\langle\psi, \rho_{\mathbb{V}}^{\text {group }}\left(\widetilde{g}_{\underline{\ell}, \underline{P}}\right) v^{\prime}\right\rangle\right\} .
$$


Using the expression (20) for $T(z)$, and the fact that $\left(\mathrm{id} \otimes \rho_{\mathbb{W}}\right)\left(\ell_{\underline{\ell}}^{i n}(z)\right)(v)=0$, we find

$$
\begin{aligned}
& 2 \kappa\left\langle\psi, \rho_{\mathbb{W}}^{\text {group }}\left(\widetilde{g}_{\underline{\ell}, \underline{P}}\right) \rho_{\mathbb{W}}(T(z)) v_{0, k}\right\rangle \\
& =\left\langle\psi, \rho_{\mathbb{W}}^{\text {group }}\left(\widetilde{g}_{\underline{\ell}, \underline{P}}\right) \rho_{\mathbb{W}}\left[\sum_{\alpha} \ell_{\underline{\ell}, x_{\alpha}}^{\text {out }}(z) \ell_{y_{\alpha}}(z)+\sum_{\alpha} a_{x_{\alpha}, \underline{P}}(\underline{\ell}, z) \ell_{y_{\alpha}}(z)\right] v\right\rangle+s_{\underline{P}}(\underline{\ell}, z) f_{\psi}(\underline{\ell} \mid v),
\end{aligned}
$$

where we set $\ell_{\underline{\ell}, x}(z)=\left\langle x \otimes \mathrm{id} \otimes \mathrm{id}, \ell_{\underline{\ell}}(z)\right\rangle_{\overline{\mathfrak{g}}}, \ell_{\underline{\ell}, x}^{\text {out }}(z)=\left\langle x \otimes \mathrm{id} \otimes \mathrm{id}, \ell_{\underline{\ell}}^{\text {out }}(z)\right\rangle_{\overline{\mathfrak{g}}} a_{x, \underline{P}}(\underline{\ell}, z)=$ $\left\langle x \otimes \mathrm{id}, a_{\underline{P}}(\underline{\ell}, z),\right\rangle_{\overline{\mathfrak{g}}}$, for $x$ in $\overline{\mathfrak{g}}$.

Let us set, for $x$ in $\overline{\mathfrak{g}}, \ell_{\underline{P}, x}^{\text {diff }}(z)=\left\langle x \otimes \mathrm{id} \otimes \mathrm{id}, \ell_{\underline{P}}^{\text {diff }}(z)\right\rangle_{\overline{\mathfrak{g}}} \cdot \ell_{x, u}^{\text {diff }}(z)$ belongs then to $\mathbb{C}\left[\ell_{i}, \partial_{\ell_{i}}, i=1, \ldots, 3 g\right]\left[\overline{\operatorname{Den}}(\underline{P}, \underline{\ell})^{-1}\right] \otimes \Omega(C)$. Lemma 5.5 then implies that

$$
\begin{aligned}
& 2 \kappa\left\langle\psi, \rho_{\mathbb{W}}^{\text {group }}\left(\widetilde{g}_{\underline{\ell}, \underline{P}}\right) \rho_{\mathbb{W}}(T(z)) v\right\rangle \\
& =\sum_{\alpha}\left[\ell_{\underline{P}, x_{\alpha}}^{\text {diff }}(z)+a_{\underline{P}, x_{\alpha}}(\underline{\ell}, z)\right]\left\{\left\langle\psi, \rho_{\mathbb{W}}\left[\ell_{\underline{\ell}}^{\text {out }}(z)\right] v\right\rangle\right\}+s_{\underline{P}}(\underline{\ell}, z) f_{\psi}(\underline{\ell}, \underline{P} \mid v) \\
& =\left\{\left[\sum_{\alpha} \ell_{\underline{P}, x_{\alpha}}^{\text {diff }}(z) \ell_{\underline{P}, y_{\alpha}}^{\text {diff }}(z)+\sum_{\alpha} a_{\underline{P}, x_{\alpha}}(\underline{\ell}, z) \ell_{\underline{P}, y_{\alpha}}^{\text {diff }}(z)+s_{\underline{P}}(\underline{\ell}, z)\right] f_{\psi}\right\}(\underline{\ell}, \underline{P} \mid v) .
\end{aligned}
$$

We have therefore

Theorem 5.1. Let us set

$$
T_{\underline{P}}^{\text {diff }}(z)=\sum_{\alpha} \ell_{\underline{P}, x_{\alpha}}^{\text {diff }}(z) \ell_{\underline{P}, y_{\alpha}}^{\text {diff }}(z)+\sum_{\alpha} a_{\underline{P}, x_{\alpha}}(\underline{\ell}, z) \ell_{\underline{P}, y_{\alpha}}^{\text {diff }}(z)+s_{\underline{P}}(\underline{\ell}, z) .
$$

Then $T_{\underline{P}}^{\text {diff }}(z)$ belongs to $\mathbb{C}\left[\ell_{i}, \partial_{\ell_{i}}, i=1, \ldots, 3 g\right]\left[\operatorname{Den}(\underline{P}, \underline{\ell})^{-1}\right] \otimes H^{0}\left(C, \Omega_{C}^{2}\left(2 \sum_{i=1}^{3 g} P_{i}\right)\right)$. In other words, it is a quadratic differential in $z$, regular on $C$ except for double poles at the $P_{i}, i=1, \ldots, 3 g$, with coefficients differential operators in $\underline{\ell}$.

There are differential operators $T_{\underline{P}, i}^{\text {diff }}$ in $\underline{\ell}$, polynomial in $k$ of degree $\leq 1$, such that the expansion of $T_{\underline{P}}^{\text {diff }}(z)$ for $z$ near $P_{i}$ is

$$
T_{\underline{P}}^{d i f f}(z)=2 \kappa k\left(\frac{1}{2} \frac{d z_{P_{i}}}{z_{P_{i}}}\right)^{2}+\kappa T_{\underline{P}, i}^{d i f f} \frac{\left(d z_{P_{i}}\right)^{2}}{z_{P_{i}}}+O(1) .
$$

For any $\mathfrak{g}^{\text {out }, \text { ext }}$-invariant form $\psi$ on $\mathbb{W}$, we have

$$
\left\langle\psi, \rho_{\mathbb{W}}^{\text {group }}\left(\widetilde{g}_{\underline{\ell}, \underline{P}}\right) \rho_{\mathbb{W}}(T(z)) v\right\rangle=\frac{1}{2 \kappa}\left(T_{\underline{P}}^{\text {diff }}(z) f_{\psi}\right)(\underline{\ell} \mid v) .
$$

Proof of Thm. (23) follows from the above computations. Let us now show the properties of $T_{\underline{P}}^{\text {diff }}(z)$. We will use the following Lemma

Lemma 5.6. Let us set

$$
\begin{aligned}
& \widetilde{T}_{\underline{P}}^{\text {diff }}(z)=T_{\underline{P}}^{\text {diff }}(z)+\kappa \frac{d b_{\underline{P}}}{b_{\underline{P}}}(z) \ell_{\underline{P}, h}^{\text {diff }}(z) \\
& +2 \kappa\left[\sum_{i} \ell_{i}^{-1}\left\{d\left(d_{i, \underline{P}}\right)-d_{i, \underline{P}} \frac{d b_{\underline{P}}}{b_{\underline{P}}}\right\}(z)\right] \ell_{\underline{P}, e}^{d i f f}(z)+2 k \kappa\left(\frac{1}{2} \frac{d b_{\underline{P}}}{b_{\underline{P}}}\right)^{2}(z) .
\end{aligned}
$$


Then we have

$$
2 \kappa\left\langle\psi, \rho_{\mathbb{W}}(T(z)) \rho_{\mathbb{W}}^{\text {group }}\left(\widetilde{g}_{\underline{\ell}, \underline{P}}\right) v\right\rangle=\left(\widetilde{T}_{\underline{P}}^{\text {diff }}(z) f_{\psi}\right)(\underline{\ell}, \underline{P} \mid v) .
$$

Let $k$ be an arbitrary complex number and let $\widetilde{W}$ be any $\overline{\mathfrak{g}}$-module. Define $\widetilde{\mathbb{W}}$ as the Weyl module over $U \mathfrak{g}$ equal to $U \mathfrak{g} \otimes_{U \mathfrak{g}_{+}} \widetilde{W}$ (see sect. 4.2). Let us denote by $\rho_{\widetilde{\mathbb{W}}}$ the corresponding algebra map from $U \mathfrak{g}$ to $\operatorname{End}(\widetilde{\mathbb{W}})$. For $v$ in $\widetilde{W}$, we denote also by $v$ the vector $1 \otimes v$ of $\widetilde{\mathbb{W}}$.

Let us fix $\underline{\ell}_{0}=\left(\ell_{i, 0}\right)_{i=1, \ldots, 3 g}$ in $\left(\mathbb{C}^{\times}\right)^{3 g}$, such that $\operatorname{Den}\left(\underline{P}, \underline{\ell}_{0}\right) \neq 0$. Consider $\underline{\ell}$ as a formal variable near $\underline{\ell}_{0}$ and let us define $\gamma_{\underline{\ell}_{0}, \underline{\ell}, \underline{P}}$ as the element of $U \mathfrak{g}\left[\left[\underline{\ell}-\underline{\ell}_{0}\right]\right]$ equal to

$$
\gamma_{\underline{\ell}_{0}, \underline{\ell}, \underline{P}}=\exp \left(e\left[\sum_{i}\left(\ell_{i, 0}^{-1}-\ell_{i}^{-1}\right) d_{i, \underline{P}}\right]\right) z\left(\prod_{i}\left(\ell_{i} / \ell_{i, 0}\right)^{k}\right) .
$$

When $k$ is an integer $\geq 0$, we have $\widetilde{g}_{\underline{P}, \underline{\ell}_{0}}^{-1} \widetilde{g}_{\underline{P}, \underline{\ell}}=\gamma_{\underline{\ell}_{0}, \underline{\ell}, \underline{P}}$. Then the analogue of Lemma 5.6 and of formula (23) for the Weyl module $\widetilde{\mathbb{W}}$ are:

Lemma 5.7. Let $\psi_{0}$ be a $\operatorname{Ad}\left(g_{\underline{P}, \underline{\ell}_{0}}\right)\left(\mathfrak{g}^{\text {out }}\right)$-invariant form on $\widetilde{\mathbb{W}}$. For any $v^{\prime}$ in $\widetilde{\mathbb{W}}$, $\left\langle\psi_{0}, \rho_{\widetilde{\mathbb{W}}}\left[\operatorname{Ad}\left(g_{\underline{P}, \underline{\ell}_{0}}^{-1}\right)(T(z)) \gamma_{\underline{\underline{\ell}}_{0}, \underline{\ell}, \underline{P}}\right]\left(v^{\prime}\right)\right\rangle$ and $\left\langle\psi_{0}, \rho_{\widetilde{\mathbb{W}}}\left[\gamma_{\underline{\ell}_{0}, \underline{\ell}, \underline{P}}\right]\left(v^{\prime}\right)\right\rangle$ are formal functions in $\underline{\ell}-\underline{\ell}_{0}$, and we have, for any $v$ in $\widetilde{W}$,

$$
\left\langle\psi_{0}, \rho_{\widetilde{\mathbb{W}}}\left[\operatorname{Ad}\left(g_{\underline{P}, \underline{\ell}_{0}}^{-1}\right)(T(z)) \gamma_{\underline{\ell}_{0}, \underline{\ell}, \underline{P}}\right](v)\right\rangle=\widetilde{T}_{\underline{P}}^{\text {diff }}(z)\left\{\left\langle\psi_{0}, \rho_{\widetilde{\mathbb{W}}}\left[{\underline{\underline{\ell}_{0}, \underline{\ell}, \underline{P}}}\right](v)\right\rangle\right\}
$$

and

$$
\left\langle\psi_{0}, \rho_{\widetilde{\mathbb{W}}}\left[\gamma_{\underline{\ell}_{0}, \underline{\ell}, \underline{P}} T(z)\right](v)\right\rangle=T_{\underline{P}}^{\text {diff }}(z)\left\{\left\langle\psi_{0}, \rho_{\widetilde{\mathbb{W}}}\left[\gamma_{\underline{\ell}_{0}, \underline{\ell}, \underline{P}}\right](v)\right\rangle\right\}
$$

(equalities in $\mathbb{C}\left[\left[\underline{\ell}-\underline{\ell}_{0}\right]\right]$ ).

The interest of identities (25) and (26) lies in the fact that any formal function of $\underline{\ell}-\underline{\ell}_{0}$ is of the form $\left\langle\psi_{0}, \rho_{\widetilde{\mathbb{W}}}\left[\gamma_{\underline{\ell}_{0}, \underline{\ell}, \underline{P}}\right](v)\right\rangle$. More precisely, we have:

Lemma 5.8. The correlation functions map $\psi_{0} \mapsto\left(v \mapsto\left\langle\psi_{0}, \rho_{\widetilde{\mathbb{W}}}\left[\gamma_{\underline{\ell}_{0}, \underline{\ell}, \underline{P}}\right](v)\right\rangle\right) d e$ fines an isomorphism from the space of $\operatorname{Ad}\left(g_{\underline{P}, \underline{\ell}_{0}}\right)\left(\mathfrak{g}^{\text {out }}\right)$-invariant forms on $\widetilde{\mathbb{W}}$ to $\operatorname{Hom}_{\overline{\mathfrak{g}}}\left(\widetilde{\mathbb{W}}, \mathbb{C}\left[\left[\underline{\ell}-\underline{\ell}_{0}\right]\right]\right)$.

Here $\mathbb{C}\left[\left[\underline{\ell}-\underline{\ell}_{0}\right]\right]$ is endowed with the action of $\overline{\mathfrak{g}}$ defined by $e \mapsto \sum_{i} \frac{\partial}{\partial \ell_{i}}, h \mapsto$ $\sum_{i}-\left(2 \ell_{i} \frac{\partial}{\partial \ell_{i}}+k\right), f \mapsto \sum_{i}-\left(\ell_{i}^{2} \frac{\partial}{\partial \ell_{i}}+k \ell_{i}\right)$.

Lemma 5.8 follows from the fact that the map

$$
U \mathfrak{g}_{\underline{\ell}}^{\text {out }} \otimes \mathbb{C}\left[e\left[d_{i, \underline{P}}\right]\right] \otimes U(\overline{\mathfrak{g}}[[t]]) \rightarrow U L \mathfrak{g},
$$

induced by the multiplication, is surjective, and its kernel is generated by the relations

$$
e[1]-\sum_{i} \ell_{i}^{2} e\left[d_{i, \underline{P}}\right] \in \mathfrak{g}_{\underline{\ell}}^{\text {out }}, h[1]-\sum_{i} 2 \ell_{i} e\left[d_{i, \underline{P}}\right] \in \mathfrak{g}_{\underline{\ell}}^{\text {out }}, f[1]+\sum_{i} e\left[d_{i, \underline{P}}\right] \in \mathfrak{g}_{\underline{\ell}}^{\text {out }} .
$$


Taking for example $\widetilde{W}=\mathbb{C}\left[\left[\underline{\ell}-\underline{\ell}_{0}\right]\right]$, we see that any element of $\mathbb{C}\left[\left[\underline{\ell}-\underline{\ell}_{0}\right]\right]$ can be obtained as a $\left\langle\psi_{0}, \rho_{\widetilde{\mathbb{W}}}\left[\gamma_{\ell_{0}, \underline{\ell}, \underline{P}}\right](v)\right\rangle$.

For any vector field $\xi$ regular on $C \backslash\left\{P_{0}\right\}, T[\xi]=\operatorname{res}_{P_{0}}(\xi(z) T(z))$ belongs to $\mathfrak{g}^{\text {out }} U \mathfrak{g}$ (see [6]). It follows that the right side of (25) is a quadratic differential in $z$, regular outside $P_{0}$.

It follows that $\widetilde{T}_{\underline{P}}^{\text {diff }}(z)$ is a quadratic differential in $z$, regular outside $P_{0}$, with coefficients differential operators in $\underline{\ell}$. Moreover, formula (21) expressing $T^{\text {diff }}(z)$ implies that $T^{\text {diff }}(z)$ is regular at $z=P_{0}$.

The other functional properties of $T^{\operatorname{diff}}(z)$ follow from formula (24) expressing $T^{\operatorname{diff}}(z)$ in term of $\widetilde{T}^{\text {diff }}(z)$ :

$-d \log b_{\underline{P}}$ and $\ell_{\underline{P}}^{\text {diff }}$ are univalued differentials in $z$, therefore $T^{\operatorname{diff}}(z)$ is a univalued quadratic differential;

- the expansions

$$
\ell_{\underline{P}, h}^{d i f f}(z)=2 \frac{d z_{P_{i}}}{z_{P_{i}}}\left(-\ell_{i} \partial_{\ell_{i}}+\frac{k}{2}\right)+O(1), \quad \ell_{\underline{P}, e}^{d i f f}(z)=\frac{d z_{P_{i}}}{z_{P_{i}}}\left(-\ell_{i}^{2} \partial_{\ell_{i}}+k \ell_{i}\right)+O(1),
$$

and $s_{\underline{P}}(z)=\left(\frac{d z_{P_{i}}}{z_{P_{i}}}\right)^{2}+O\left(z_{P_{i}}^{-1}\right)$ for $z$ near $P_{i}$ imply that $T^{\text {diff }}(z)$ has the expansion

$$
T^{\text {diff }}(z)=\frac{1}{2} \kappa k\left(\frac{d z_{P_{i}}}{z_{P_{i}}}\right)^{2}+O\left(z_{P_{i}}^{-1}\right)
$$

for $z$ near $P_{i}$. Expansions (22) follow. Since the coefficients of $\frac{\left(d z_{P_{i}}\right)^{2}}{z_{P_{i}}}$ in these expansions are the same as those of $\left(T^{\text {diff }}-\widetilde{T}^{\text {diff }}\right)(z)$, which are given by formula (24), they are polynomial in $k$, of degree $\leq 1$.

Proof of Lemma 5.6. Let us denote by $(U \mathfrak{g})_{k}$ the quotient of $U \mathfrak{g}$ by the ideal generated by $K-k \cdot 1$, and by $(U \mathfrak{g})_{k, \text { compl }}$ the completion of $(U \mathfrak{g})_{k}$ w.r.t. the topology defined by the right ideals generated by the $x\left[z^{l}\right], l \geq N$ (the Laurent coefficients of $T(z)$ belong to $(U \mathfrak{g})_{k, \text { compl }}$.)

Then $\widehat{G(\mathcal{K})}$ acts on $(U \mathfrak{g})_{k, c o m p l}$ by the adjoint action; this action factors through $G(\mathcal{K})$; so we have a sequence of group morphisms

$$
\widehat{G(\mathcal{K})} \rightarrow G(\mathcal{K}) \rightarrow \text { Aut }_{\text {algebra }}\left[(U \mathfrak{g})_{k, \text { compl }}\right]
$$

We denote by Ad any of the maps $G(\mathcal{K}) \rightarrow \operatorname{Aut}_{\mathbb{C}}\left[(U \mathfrak{g})_{k, \text { compl }}\right]$ and $\widehat{G(\mathcal{K})} \rightarrow$ $\operatorname{Aut}_{\mathbb{C}}\left[(U \mathfrak{g})_{k, \text { compl }}\right]$.

Moreover, it follows from the formula

$$
[T(z), x[f]]=-(d f)(z) x(z)
$$

for $x$ in $\overline{\mathfrak{g}}$, that if $f$ belongs to $\mathcal{K}$,

$$
\operatorname{Ad}\left(\left(\begin{array}{ll}
1 & f \\
0 & 1
\end{array}\right)\right)[T(z)]=T(z)+(d f)(z) e(z)
$$


and that for $\phi$ in $\mathbb{C}\left[\left[z_{P_{0}}\right]\right]^{\times}$,

$$
\operatorname{Ad}\left(\left(\begin{array}{cc}
\phi & 0 \\
0 & \phi^{-1}
\end{array}\right)\right)[T(z)]=T(z)+\frac{d \phi}{\phi}(z) h(z)+K\left(\frac{d \phi}{\phi}\right)^{2}(z) .
$$

Moreover, we have also

$$
\operatorname{Ad}\left(T_{\widehat{G(\mathcal{K})}}\right)[T(z)]=T(z)+\frac{1}{2} \frac{d z_{P_{0}}}{z_{P_{0}}}(z) h(z)+K\left(\frac{1}{2} \frac{d z_{P_{0}}}{z_{P_{0}}}\right)^{2}(z) .
$$

Let then $\psi$ belong to $\mathcal{K}^{\times}$; let us denote $v(\psi)$ the valuation of $\psi$ at $P_{0}$, and set $t[\psi]=T_{\widehat{G(\mathcal{K})}}^{v(\psi)}\left(\begin{array}{cc}\psi_{0}^{1 / 2} & 0 \\ 0 & \left(\psi_{0}^{1 / 2}\right)^{-1}\end{array}\right)$, where $\psi_{0}=z_{P_{0}}^{-v(\psi)} \psi$ and $\psi_{0}^{1 / 2}$ is any square root of $\psi_{0}$, we have therefore

$$
\operatorname{Ad}(t[\psi])(T(z))=T(z)+\frac{1}{2} \frac{d \psi}{\psi}(z) h(z)+K\left(\frac{1}{2} \frac{d \psi}{\psi}\right)^{2}(z) .
$$

We have therefore

$$
\begin{aligned}
& \operatorname{Ad}\left(\widetilde{g}_{\underline{\ell}, \underline{P}}\right)^{-1}(T(z))=\operatorname{Ad}\left(\iota\left(\left(\begin{array}{cc}
1 & \sum_{i} \ell_{i}^{-1} d_{i, \underline{P}} \\
0 & 1
\end{array}\right)\right)\right)\left[T(z)+\frac{1}{2} \frac{d b_{\underline{P}}}{b_{\underline{P}}}(z) h(z)+K\left(\frac{1}{2} \frac{d b_{\underline{P}}}{b_{\underline{P}}}\right)^{2}(z)\right] \\
& =T(z)+\left[\sum_{i} \ell_{i}^{-1}\left\{d\left(d_{i, \underline{P}}\right)-d_{i, \underline{P}} \frac{d b_{\underline{P}}}{b_{\underline{P}}}\right\}(z)\right] e(z)+\frac{1}{2} \frac{d b_{\underline{P}}}{b_{\underline{P}}}(z) h(z)+K\left(\frac{1}{2} \frac{d b_{\underline{P}}}{b_{\underline{P}}}\right)^{2}(z) .
\end{aligned}
$$

So

$$
\begin{aligned}
& 2 \kappa\left\langle\psi, \rho_{\mathbb{W}}(T(z)) \rho_{\mathbb{W}}^{\text {group }}\left(\widetilde{g}_{\underline{\ell}, \underline{P}}\right) v\right\rangle \\
& =2 \kappa\left\langle\psi, \rho_{\mathbb{W}}^{\text {group }}\left(\widetilde{g}_{\underline{\ell}, \underline{P}}\right) \rho_{\mathbb{W}}(T(z)) v\right\rangle \\
& +2 \kappa\left[\sum_{i} \ell_{i}^{-1}\left\{d\left(d_{i, \underline{P}}\right)-d_{i, \underline{P}} \frac{d b_{\underline{P}}}{b_{\underline{P}}}\right\}(z)\right]\left\langle\psi, \rho_{\mathbb{W}}^{\text {group }}\left(\widetilde{g}_{\underline{\ell}, \underline{P}}\right) \rho_{\mathbb{W}}(e(z)) v\right\rangle \\
& +2 \kappa \frac{1}{2} \frac{d b_{\underline{P}}}{b_{\underline{P}}}(z)\left\langle\psi, \rho_{\mathbb{W}}^{\text {group }}\left(\widetilde{g}_{\underline{\underline{\ell}}, \underline{P}}\right) \rho_{\mathbb{W}}(h(z)) v\right\rangle \\
& +2 \kappa k\left(\frac{1}{2} \frac{d b_{\underline{P}}}{b_{\underline{P}}}\right)^{2}(z)\left\langle\psi, \rho_{\mathbb{W}}^{\text {group }}\left(\widetilde{g}_{\underline{\ell}, \underline{P}}\right) v\right\rangle .
\end{aligned}
$$

On the other hand, we have for any $x$ in $\overline{\mathfrak{g}}$,

$$
\left\langle\psi, \rho_{\mathbb{W}}^{\text {group }}\left(\widetilde{g}_{\underline{\ell}, \underline{P}}\right) \rho_{\mathbb{W}}(x(z)) v\right\rangle=\left(\ell_{\underline{P}, x}^{\text {diff }}(z) f_{\psi}\right)(\underline{\ell}, \underline{P} \mid v) .
$$

Lemma 5.6 follows.

\section{Explicit Form of the Beilinson-Drinfeld operators}

6.1. Pull-back of the det line bundle. Recall that the line bundle det is defined over $\operatorname{Bun}_{2}(C)_{\mathcal{O}\left(\sum_{i} P_{i}\right)}$ as $\operatorname{det} R \Gamma$ ([2]).

Proof of Prop. 1.2. A line bundle over $\left(\mathbb{C} P^{1}\right)^{N}$ is necessarily of the form $\mathcal{O}\left(k_{1}\right) \otimes \cdots \otimes \mathcal{O}\left(k_{N}\right)$. To determine the $k_{i}$, let us compute the restriction of $\mathcal{H}_{\underline{P}}^{*}($ det $)$ 
HECKE-TYURIN PARAMETRIZATION OF THE HITCHIN AND KZB SYSTEMS

to $\left(\ell_{1}, \ldots, \ell_{i-1}\right) \times \mathbb{C} P^{1} \times\left(\ell_{i+1}, \ldots, \ell_{N}\right)$, for generic $\ell_{i}$. For $\underline{\ell}$ generic, the fiber of $\mathcal{H}_{\underline{P}}^{*}($ det $)$ at $\underline{\ell}$ is the determinant of $\left\{\left(\alpha_{i}\right) \in \mathbb{C}^{3 g} \mid \sum_{i} \alpha_{i} \omega_{a}\left(P_{i}\right)=\sum_{i} \alpha_{i} \ell_{i} \omega_{a}\left(P_{i}\right)=\right.$ $0\}$, which we view as

$$
\left\{\left(\alpha_{j}, \alpha_{i}^{\prime}\right)_{j \neq i} \in \mathbb{C}^{3 g} \mid \sum_{j \neq i} \alpha_{j} \omega_{a}\left(P_{j}\right)+\alpha_{i}^{\prime} \ell_{i}^{-1} \omega_{a}\left(P_{i}\right)=\sum_{j \neq i} \alpha_{j} \ell_{j} \omega_{a}\left(P_{j}\right)+\alpha_{i}^{\prime} \omega_{a}\left(P_{i}\right)=0\right\}
$$

in the neighborhood of $\ell_{i}=\infty$. It follows that the restriction of $\mathcal{H}_{\underline{P}}^{*}$ (det) to $\left(\ell_{1}, \ldots, \ell_{i-1}\right) \times \mathbb{C} P^{1} \times\left(\ell_{i+1}, \ldots, \ell_{N}\right)$ is isomorphic to $\mathcal{O}(1)$, therefore all $k_{i}$ are equal to 1.

6.2. Explicit form of the Beilinson-Drinfeld operators. The BeilinsonDrinfeld operators are differential operators acting on sections of $\operatorname{det}^{-2}$. They are defined as the action on $\operatorname{Bun}_{2}(C)_{\mathcal{O}\left(\sum_{i} P_{i}\right)}$ of the central elements of the critical level quotient $U \mathfrak{g} /(K+2 \cdot 1)$ of the enveloping algebra of $\mathfrak{g}$. A generating series for these central elements is the Sugawara series $T(z)(d z)^{2}=\sum_{n} T_{n} z^{-n-2}(d z)^{2}$; let $T_{n}^{(B D)}$ be the action of $T_{n}$ by differential operators. Then the series $\sum_{n} T_{n}^{(B D)} z^{-n-2}(d z)^{2}$ is a linear combination of the quadratic differentials on $C$. Let $\left(\omega_{\alpha}^{(2)}\right)_{a=1, \ldots, 3 g-3}$ be a basis of $H^{0}\left(C, \Omega_{C}^{\otimes 2}\right)$ and let us set $\sum_{n} T_{n}^{(B D)} z^{-n-2}(d z)^{2}=\sum_{\alpha} T_{\alpha}^{B D} \omega_{\alpha}^{(2)}$. Then the $T_{n}^{(B D)}$ are all linear combinations of the $T_{\alpha}^{B D}$.

Proof of Thm. 1.g The expansions (22) imply that $T^{\text {diff }}(z)$ has no poles at the $P_{i}$. The fact that the operators $T_{P, \alpha}^{\text {diff }}$ commute follows from formula (26), the fact that any formal series in $\mathbb{C}\left[\left[\underline{\underline{\ell}}-\underline{\ell}_{0}\right]\right]$ can be obtained as a correlation function $\left\langle\psi_{0}, \rho\left[\gamma_{\underline{\ell}_{0}, \underline{\ell}, \underline{P}}\right](v)\right\rangle$, and the commutativity of the components of the Sugawara tensor when $\kappa=0$.

\section{ExPliCit FORM OF THE KZB CONNECTION}

7.1. Dependence of $i_{P}(\psi)$ in $\underline{P}$. Let us denote by $\left(C^{3 g}\right)_{P_{0}}$ the subspace of $\left(C \backslash\left\{P_{0}\right\}\right)^{3 g}$, formed of the systems $\underline{P}=\left(P_{i}\right)_{i=1, \ldots, 3 g}$, such that $\sum_{i} A\left(P_{i}\right)=$ $3 g A\left(P_{0}\right)$.

Let us denote by $i_{\underline{P}}$ the linear map

$$
i_{\underline{P}}:\left(\mathbb{W}^{*}\right)^{\mathfrak{g}^{\text {out }}} \rightarrow \operatorname{Hom}_{\overline{\mathfrak{g}}}\left(W, \otimes_{i=1}^{3 g} \mathbb{C}\left[\ell_{i}\right]_{\leq k}\right),
$$

indexed by $\underline{P}$ in $\left(C^{3 g}\right)_{P_{0}}$ : we have $i_{\underline{P}}(\psi)=\left[v \mapsto f_{\psi}(\underline{\ell}, \underline{P} \mid v)\right]$. In this section, we describe the dependence of $i_{P}(\psi)$ on $\underline{P}$, when $\psi$ is fixed. For this, we first compare the maps $\mathcal{H}_{\underline{P}}$ and $\mathcal{H}_{\underline{P}^{\prime}}$, for $\underline{P}^{\prime}$ infinitely close to $\underline{P}$.

7.1.1. Infinitesimal comparison of $\mathcal{H}_{\underline{P}}$ and $\mathcal{H}_{\underline{P}^{\prime}}$. Let us fix variations $\delta P_{i}$ of the points $P_{i}$, such that $\delta\left(\sum_{i} A\left(P_{i}\right)\right)=0$. Let us fix $\underline{\ell}$ such that $\operatorname{Den}(\underline{P}, \underline{\ell}) \neq 0$. It follows from Thm. 1.1 that one can find $\underline{\ell}^{\prime}$ infinitely close to $\underline{\ell}$, such that $\mathcal{H}_{\underline{P}}(\underline{\ell})=\mathcal{H}_{\underline{P}^{\prime}}\left(\underline{\ell}^{\prime}\right)$. In this section, we compute the variation $\delta \underline{\ell}=\underline{\ell}^{\prime}-\underline{\ell}$. 
Let us denote by $M(z)$ the matrix corresponding to the isomorphism from $\mathcal{H}\left(\underline{P}^{\prime}, \underline{\ell}^{\prime}\right)$ to $\mathcal{H}(\underline{P}, \underline{\ell})$. We have

$$
M(z)=\operatorname{id}_{\mathbb{C}^{2}}+\sum_{i}\left(\begin{array}{l}
1 \\
\ell_{i}
\end{array}\right)\left(\begin{array}{ll}
\alpha_{i} & \beta_{i}
\end{array}\right) r^{\left(P_{i}\right)}(z)
$$

where $\alpha_{i}=\alpha_{i}^{(1)}+\alpha_{i}^{(2)}+\ldots$ and $\beta_{i}=\beta_{i}^{(1)}+\beta_{i}^{(2)}+\ldots$ are infinitesimal vectors at $P_{i}\left(\alpha_{i}^{(1)}\right.$ is the linear in $\delta P_{i}$ part of $\alpha_{i}, \alpha_{i}^{(2)}$ the quadratic part, etc.), satisfying the equations

$$
\sum_{i} \omega_{a}\left(P_{i}\right)\left(\begin{array}{l}
1 \\
\ell_{i}
\end{array}\right)\left(\begin{array}{ll}
\alpha_{i} & \beta_{i}
\end{array}\right)=0
$$

for each $a$, and $M\left(P_{i}+\delta P_{i}\right)\left(\begin{array}{c}1 \\ \ell_{i}+\delta \ell_{i}\end{array}\right)=0$, for any $i$, which implies at first order $\left(\begin{array}{c}1 \\ \ell_{i}+\delta \ell_{i}\end{array}\right)+\sum_{j \neq i} r^{\left(P_{j}\right)}\left(P_{i}\right)\left(\alpha_{j}^{(1)}+\ell_{i} \beta_{j}^{(1)}\right)\left(\begin{array}{l}1 \\ \ell_{j}\end{array}\right)+\left[r^{\left(P_{i}\right)}\left(P_{i}+\delta P_{i}\right)\left(\alpha_{i}^{(1)}+\ell_{i} \beta_{i}^{(1)}\right)+\kappa_{i}\right]\left(\begin{array}{l}1 \\ \ell_{i}\end{array}\right)=0$, where we set $\kappa_{i}=r^{\left(P_{i}\right)}\left(P_{i}+\delta P_{i}\right)\left(\alpha_{i}^{(2)}+\ell_{i} \beta_{i}^{(2)}\right)$.

The main terms of the left side of this equation should cancel, therefore

$$
\beta_{i}^{(1)}=-\frac{1}{\ell_{i}}\left(\alpha_{i}^{(1)}+\delta P_{i}\right)
$$

(27) then yields the system

$$
\sum_{i} \omega_{a}\left(P_{i}\right) \alpha_{i}^{(1)}=0, \quad \sum_{i} \omega_{a}\left(P_{i}\right) \ell_{i} \alpha_{i}^{(1)}=0, \quad \sum_{i} \omega_{a}\left(P_{i}\right) \ell_{i}^{-1}\left[\alpha_{i}^{(1)}+\delta P_{i}\right]=0,
$$

as well as the equation $\sum_{i} \omega_{a}\left(P_{i}\right) \delta P_{i}=0$ for any $a=1, \ldots, g$, which is satisfied because $\delta\left(\sum_{i} A\left(P_{i}\right)\right)=0$. We then get

$$
\kappa_{i}=-\sum_{j \neq i} r^{\left(P_{j}\right)}\left(P_{i}\right)\left(\alpha_{j}^{(1)}+\ell_{i} \beta_{j}^{(1)}\right)
$$

and

$$
\delta \ell_{i}=-\sum_{j \neq i} r^{\left(P_{j}\right)}\left(P_{i}\right) \ell_{j}\left(\alpha_{j}^{(1)}+\ell_{i} \beta_{j}^{(1)}\right)-\kappa_{i} \ell_{i}=\sum_{j \neq i} r^{\left(P_{j}\right)}\left(P_{i}\right) \ell_{i j}\left(\alpha_{j}^{(1)}+\ell_{i} \beta_{j}^{(1)}\right) .
$$

(28) yields

$$
\alpha_{i}^{(1)}=\frac{1}{\operatorname{Den}(\underline{P}, \underline{\ell})} \sum_{j}-\left(\ell_{i} / \ell_{j}\right) \delta P_{j} \operatorname{Den}(\underline{P}, \underline{\ell})_{\mid \ell_{i}=0, P_{i} \rightarrow P_{j}} .
$$

It follows that

$$
\delta \ell_{i}=\frac{1}{\operatorname{Den}(\underline{P}, \underline{\ell})} \sum_{j \neq i} r^{\left(P_{j}\right)}\left(P_{i}\right) \ell_{i j}^{2} \sum_{l} \frac{1}{\ell_{l}} \delta P_{l} \operatorname{Den}(\underline{P}, \underline{\ell}) \mid \ell_{j}=0, P_{j} \rightarrow P_{l}-\sum_{j \neq i} r^{\left(P_{j}\right)}\left(P_{i}\right) \ell_{i j} \frac{\ell_{i}}{\ell_{j}} \delta P_{j} .
$$


HECKE-TYURIN PARAMETRIZATION OF THE HITCHIN AND KZB SYSTEMS

7.1.2. Dependence of $i_{\underline{P}}(\psi)$ on $\underline{P}$. Let us preserve the notation of the previous section.

Let us set

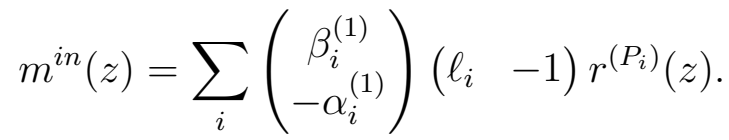

The Laurent expansion of $m^{i n}(z)$ at $P_{0}$ belongs to $\mathfrak{g l}_{2}\left[\left[z_{P_{0}}\right]\right]$. It follows from the previous section that there exists an element $m^{\text {out }}$ of $\mathfrak{g l}_{2} \otimes R$ such that

$$
g_{\underline{P}+\delta \underline{P}, \underline{\ell}+\delta \underline{\ell}}=\exp \left(m^{\text {out }}\right) g_{\underline{P}, \underline{\ell}} \exp \left(m^{\text {in }}\right) .
$$

Let us denote by $G(\mathcal{K}) \rtimes \mathbb{Z}$ the quotient of $\widehat{G(\mathcal{K})} \rtimes \mathbb{Z}$ by its center, and let $\widetilde{g}_{\underline{P}, \underline{\ell}}^{\prime}$ be the image of $\widetilde{g}_{\underline{P}, \underline{\ell}}$ by the projection $\widehat{G(\mathcal{K})} \rtimes \mathbb{Z} \rightarrow G(\mathcal{K}) \rtimes \mathbb{Z}$. Let $\pi$ be the projection map from $\mathfrak{g l}_{2}$ to $\mathfrak{s l}_{2}$ and set

$$
m^{\text {out }}=(\pi \otimes \mathrm{id})\left(m^{\text {out }}\right), \quad m^{\text {in }}=(\pi \otimes \mathrm{id})\left(m^{\text {in }}\right) .
$$

Then we have

$$
\widetilde{g}_{\underline{P}+\delta \underline{P}, \underline{\ell}+\delta \underline{\ell}}^{\prime}=\exp \left(m^{\text {out }}\right) \widetilde{g}_{\underline{P}, \underline{\ell}}^{\prime} \exp \left(m^{\text {in }}\right) ;
$$

the Lie algebraic meaning of this equality is that

$$
\widetilde{g}_{\underline{P}+\delta \underline{P}, \underline{\ell}+\delta \underline{\ell}}^{\prime} \widetilde{g}_{\underline{P}, \underline{\ell}}^{\prime-1}=m^{\prime \text { out }}+\widetilde{g}_{\underline{P}, \underline{\ell}}^{\prime} m^{\prime i n} \widetilde{g}_{\underline{P}, \underline{\ell}}^{\prime-1} .
$$

Let us denote by $x^{\text {in }}$ and $x^{\text {out }}$ the images of $m^{\text {in }}$ and $m^{\text {out }}$ by the map $L \overline{\mathfrak{g}} \rightarrow \mathfrak{g}$, $x \otimes f \mapsto x[f](=(x \otimes f, 0))$. Then $x^{i n}$ belongs to the linear span $\mathfrak{g}^{+, n o} K$ of the $x\left[t^{i}\right], i \geq 0, x \in \overline{\mathfrak{g}}$, and $x^{\text {out }}$ belongs to $\mathfrak{g}^{\text {out }, \text { ext }}$.

Let us denote by $\kappa$ the map from $\mathfrak{g}$ to $\mathbb{C}$ defined by $\kappa(x \otimes f, a K)=a k$.

Proposition 7.1. Let us set

$$
Z=\kappa\left\{\widetilde{g}_{\underline{P}+\delta \underline{P}, \underline{\ell}+\delta \underline{\underline{\ell}}} \widetilde{\widetilde{P}}_{\underline{P}, \underline{\ell}}^{-1}\right\}-\kappa\left\{\widetilde{g}_{\underline{P}, \underline{\ell}} x^{i n} \widetilde{g}_{\underline{P}, \underline{\ell}}^{-1}\right\} .
$$

Then we have

$$
Z=k \sum_{j \neq i} \alpha_{i}^{(1)} \frac{\ell_{i}}{\ell_{j}} r^{\left(P_{i}\right)}\left(P_{j}\right)+k \sum_{i} \frac{\delta \ell_{i}}{\ell_{i}},
$$

and

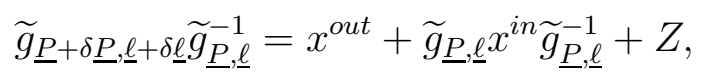

therefore

$$
\widetilde{g}_{\underline{P}+\delta \underline{P}, \underline{\ell}+\delta \underline{\ell}}=\exp \left(x^{\text {out }}\right) \widetilde{g}_{\underline{P}, \underline{\ell}} \exp \left(x^{\text {in }}\right) z(\exp (Z)),
$$

up to quadratic terms in the $\delta \ell_{i}$.

Proof. (32) follows from (30), the definition of $Z$ and the fact that $\kappa\left(x^{\text {out }}\right)=0$.

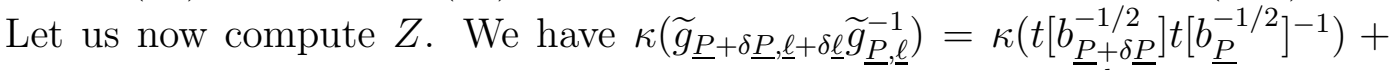
$k \sum_{i} \frac{\delta \ell_{i}}{\ell_{i}}=\kappa\left(T_{\overline{G(\mathcal{K})}}^{3 g} t\left[\left(b_{\underline{P}}^{0}+\delta \underline{P}\right)^{-1 / 2}\left(b_{\underline{P}}^{0}\right)^{1 / 2}\right] T_{\widehat{G(\mathcal{K})}}^{-3 g}\right)+k \sum_{i} \frac{\delta \ell_{i}}{\ell_{i}}=-\frac{3 g \bar{k}}{2}\left[\frac{b_{\underline{P}}+\delta \underline{P}}{b_{\underline{P}}}\left(\bar{P}_{0}\right)-1\right]+$ $k \sum_{i} \frac{\delta \ell_{i}}{\ell_{i}}$. 
On the other hand, let us set $x^{i n}=e\left[x_{e}^{i n}\right]+h\left[x_{h}^{i n}\right]++f\left[x_{f}^{i n}\right]$. We have

$$
x_{f}^{i n}=-\sum_{i} \alpha_{i}^{(1)} \ell_{i} r^{\left(P_{i}\right)}(z), \quad x_{h}^{i n}=\sum_{i} \frac{\beta_{i}^{(1)} \ell_{i}-\alpha_{i}^{(1)}}{2} r^{\left(P_{i}\right)}(z) .
$$

We have then $\kappa\left(\widetilde{g}_{\underline{P}, \underline{x}} x^{i n} \widetilde{g}_{\underline{P}, \underline{\ell}}^{-1}\right)=\kappa\left(t\left[b_{\underline{P}}^{-1 / 2}\right]\left\{h\left[x_{h}^{i n}\right]+\left[e\left[-\sum_{i} \ell_{i}^{-1} d_{i, \underline{P}}\right], f\left[x_{f}^{i n}\right]\right]\right\} t\left[b_{\underline{P}}^{-1 / 2}\right]^{-1}\right)$. After some computation, we find

$$
\kappa\left(\widetilde{g}_{\underline{P}, \underline{\underline{\ell}}} x^{i n} \widetilde{g}_{\underline{P}, \underline{\ell}}^{-1}\right)=k \sum_{j \neq i} \alpha_{i}^{(1)} \frac{\ell_{i}}{\ell_{j}} r^{\left(P_{i}\right)}\left(P_{j}\right)-\frac{3 g k}{2} \sum_{i} \delta P_{i} r^{\left(P_{i}\right)}\left(P_{0}\right) .
$$

Therefore

$$
Z=-\frac{3 g k}{2}\left[\frac{b_{P}+\delta \underline{P}}{b_{\underline{P}}}\left(P_{0}\right)-1\right]+k \sum_{j \neq i} \alpha_{i}^{(1)} \frac{\ell_{i}}{\ell_{j}} r^{\left(P_{i}\right)}\left(P_{j}\right)-\frac{3 g k}{2} \sum_{i} \delta P_{i} r^{\left(P_{i}\right)}\left(P_{0}\right)+k \sum_{i} \frac{\delta \ell_{i}}{\ell_{i}} .
$$

Since

$$
\frac{\delta b_{\underline{P}}}{b_{\underline{P}}}(z)+\sum_{i} \delta P_{i} r^{\left(P_{i}\right)}(z)
$$

vanishes for any $z$,

$$
\left[\frac{b_{\underline{P}}+\delta \underline{P}}{b_{\underline{P}}}\left(P_{0}\right)-1\right]+\sum_{i} \delta P_{i} r^{\left(P_{i}\right)}\left(P_{0}\right)=0
$$

(31) follows.

Prop. 1.4 follows from the insertion of (33) in correlation functions.

\section{Filtration of CONFORMal Blocks}

Let again $\overline{\mathfrak{g}}$ be an arbitrary semismple Lie algebra, and let $\mathbb{V}$ be an integrable module over the affine Kac-Moody algebra $\mathfrak{g}$. Let $C B(\mathbb{V})$ be the space of conformal blocks of $\mathbb{V}$. The maps $\mathcal{H}_{\underline{P}, \underline{\chi}}$ naturally lead to a filtration of $C B(\mathbb{V})$. It is defined as follows.

It follows from [3] that conformal blocks can be identified with sections of line bundles over $\operatorname{Bun}_{G}(C)$. Define $\operatorname{Fil}_{\underline{P}, \underline{\chi}}$ as the space of conformal blocks, vanishing on $\operatorname{Im} \mathcal{H}_{\underline{P}, \underline{\chi}}$. We then have a inclusions $\operatorname{Fil}_{\underline{P}, \underline{\chi}} \subset \operatorname{Fil}_{\underline{P}, \underline{\chi}^{\prime}}$ if $\chi_{i} \leq \chi_{i}^{\prime}$ for any $i$.

It is easy to define analogues $\operatorname{Fil}_{\underline{P}, \chi}, P$ of $\mathrm{Fil}_{\underline{P}, \underline{\chi}}$, which are conformal blocks vanishing on modifications of the fixed (nontrivial) bundle $P$.

Moreover, one may conjecture the following behavior of the $\mathrm{Fil}_{\underline{P}, \underline{\chi}}$ :

1) the Fil $\underline{P}, \underline{\chi}$ form a bundle over $\mathcal{M}_{g, N}$ with a flat connection; and the Fil $\underline{P}_{\underline{\underline{\chi}}, \underline{\chi}, P}$ form a bundle over the joint moduli space of triples $(C, \underline{P}, P)$ of a curve, marked points and a principal $G$-bundle over it;

2) when $P_{i}$ and $P_{j}$ both tend to a point $Q$, the limit of $\operatorname{Fil}(\underline{P}, \underline{\chi})$ is $\operatorname{Fil}\left(\underline{P}^{\prime}, \underline{\chi}^{\prime}\right)$, where $\underline{P}^{\prime}=\left(\left(P_{\alpha}\right)_{\alpha \neq i, j}, Q\right)$ and $\underline{\chi}^{\prime}=\left(\left(\chi_{\alpha}\right)_{\alpha \neq i, j}, \chi_{i}+\chi_{j}\right)$. 
It would follow from this that the dimension of $\mathrm{Fil}_{\underline{P}, \underline{\chi}, P}$ only depends on the sum $\sum_{i} \chi_{i}$, so that there is a filtration of $C B(\mathbb{V})$ by $P_{+}$. It would be interesting to see whether the formulas for the corresponding $q$-dimensions agree with those of [9, 18].

\section{REFERENCES}

[1] A. Beilinson, V. Drinfeld, Quantization of Hitchin's integrable fibration and Langlands program, Algebraic and geometric methods in mathematical physics (Kaciveli, 1993), 3-7, Math. Phys. Stud., 19, Kluwer Acad. Publ., Dordrecht, 1996.

[2] A. Beauville, Fibrés de rang 2 sur une courbe, fibré dëterminant et fonctions thêta, Bull. S.M.F., 116 (1998), 431-48.

[3] A. Beauville, Y. Laszlo, Conformal blocks and generalized theta-functions, Comm. Math. Phys. 164:2 (1994), 385-419.

[4] D. Bernard, On the WZW model for Riemann surfaces, Nucl. Phys. B, 309 (1988), 145-74.

[5] B. Enriquez, G. Felder, Commuting differential and difference operators associated with complex curves, I, math/9807145, to appear in Compositio Math.; II, math/9812152.

[6] B. Enriquez, G. Felder, On solutions of the KZB equations in genus greater than one, preprint.

[7] B. Feigin, A. Stoyanovsky, A realization of the modular functor in the space of differentials and geometric approximation of the moduli space of $G$-bundles, Funct. An. Appl. 28:1 (1994), 68-90.

[8] B. Feigin, R. Kedem, S. Loktev, T. Miwa, E. Mukhin, Combinatorics of the $\widehat{\mathfrak{s l}}_{2}$ spaces of coinvariants, math/9908003.

[9] B. Feigin, S. Loktev, On generalized Kostka polynomials and quantum Verlinde rule, math/9908003.

[10] H. Garland, The arithmetic theory of loop groups, Publ. Math. IHES 52, 5-136 (1980).

[11] K. Gawędzki, P. Tran-Ngoc-Bich, Hitchin systems at low genera, hep-th/9803101.

[12] B. van Geemen, E. Previato, On the Hitchin system, alg-geom/9410015, Duke Math. J. 85:3, 659-83 (1996).

[13] P. Grinevich, Rational solutions for the equations of commutation of differential operators, Funct. An. Appl. 16 (1982), 19-24.

[14] N. Hitchin, Stable bundles and integrable systems, Duke Math. Jour., 54 (1987), $13-52$.

[15] V. Knizhnik, A. Zamolodchikov, Current algebras and Wess-Zumino model in two dimensions, Nucl. Phys. B, 247 (1984), 83-103.

[16] S. Kumar, Infinite Grassmannian and moduli spaces of $G$-bundles, alggeom/9510016; Springer Lect. Notes in Math. (CIME), 1649 (1997), 1-49.

[17] I. Krichever, S. Novikov, Holomorphic bundles over Riemann surfaces and the KP equations, I, Funct. An. Appl. 12 (1978), 41-52.

[18] O. Foda, B. Leclerc, M. Okado, J.-Y. Thibon, Ribbon tableaux and $q$-analogues of fusion rules in WZW conformal theories, q-alg/9810008. 
[19] E. Previato, G. Wilson, Vector bundles over curves and solutions of the KP equations, Proc. Symp. Pure Math. AMS "Theta functions", Bowdoin, 49:1 (1987), 553-70.

[20] C. Seshadri, Fibrés vectoriels sur les courbes algébriques, Astérisque 96 (1962), Publ. SMF, Paris.

[21] A. Tsuchiya, Y. Ueno, Y. Yamada, Conformal field theory on universal family of stable curves with gauge symmetries, Adv. Stud. Pure Math. 10 (1989), 459-566.

[22] A. Tyurin, Classification of vector bundles over an algebraic curve of arbitrary genus, Amer. Math. Soc. Translat., II, Ser. 63 (1967), 245-79 (English); Izv. Ak. Nauk. SSSR, Ser. Mat. 29 (1965), 657-88 (Russian).

B.E.: DMA (UMR 8553 Du CNRS), ENS, 45 Rue D'Ulm, 75005 Paris, France

V.R.: Dépt. de Mathématiques, Université d’Angers, Angers, France

ITEP, 25, Bol. Cheremushinsmaya, Moscow 117259, Russia 